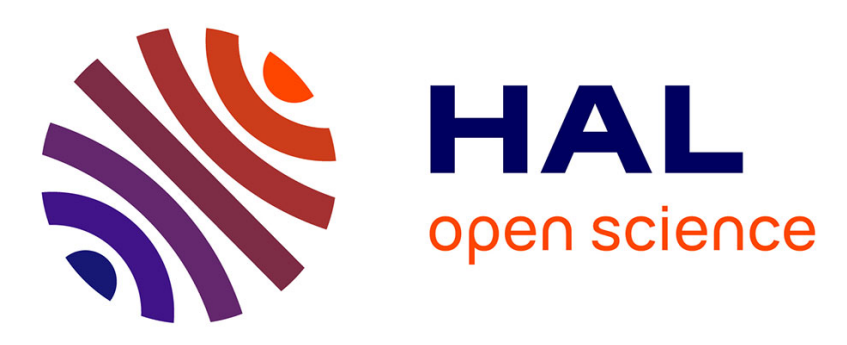

\title{
Improved resistive shunt by means of negative capacitance: new circuit, performances and multi-mode control
}

Marta Berardengo, Olivier Thomas, Christophe Giraud-Audine, Stefano

Manzoni

\section{To cite this version:}

Marta Berardengo, Olivier Thomas, Christophe Giraud-Audine, Stefano Manzoni. Improved resistive shunt by means of negative capacitance: new circuit, performances and multi-mode control. Smart Materials and Structures, 2016, 25, pp.075033. 10.1088/0964-1726/25/7/075033 . hal-01392666

\section{HAL Id: hal-01392666 https://hal.science/hal-01392666}

Submitted on 4 Nov 2016

HAL is a multi-disciplinary open access archive for the deposit and dissemination of scientific research documents, whether they are published or not. The documents may come from teaching and research institutions in France or abroad, or from public or private research centers.
L'archive ouverte pluridisciplinaire HAL, est destinée au dépôt et à la diffusion de documents scientifiques de niveau recherche, publiés ou non, émanant des établissements d'enseignement et de recherche français ou étrangers, des laboratoires publics ou privés. 


\title{
Improved resistive shunt by means of negative capacitance: new circuit, performances and multi-mode control
}

\author{
M Berardengo ${ }^{1}$, O Thomas $^{2}$, C Giraud-Audine ${ }^{3}$ and S Manzoni ${ }^{1}$ \\ ${ }^{1}$ Politecnico di Milano-Department of Mechanical Engineering, Via La Masa, 34-20156 Milan, Italy \\ ${ }^{2}$ Arts et Métiers ParisTech, LSIS UMR CNRS 7296, 8 bd. Louis XIV, 59046 Lille, France \\ ${ }^{3}$ Arts et Métiers ParisTech, L2EP, 8 bd. Louis XIV, 59046 Lille, France \\ E-mail: marta.berardengo@polimi.it
}

\begin{abstract}
This paper deals with vibration control by means of piezoelectric patches shunted with electrical impedances made up by a resistance and a negative capacitance. The paper analyses most of the possible layouts by which a negative capacitance can be built and shows that a common mathematical description is possible. This allows closed formulations to be found in order to optimise the electrical network for mono- and multi-mode control. General analytical formulations are obtained to estimate the performance of the shunt in terms of vibration reduction. In particular, it is highlighted that the main effect of a negative capacitance is to artificially enhance the electromechanical coupling factor, which is the basis of performance estimation. Stability issues relating to the use of negative capacitances are especially addressed using refined models for the piezoelectric patch capacitance. Furthermore, a new circuit based on a couple of negative capacitances is proposed and tested, showing better performances than those provided by the usual layouts with a single negative capacitance. Finally, guidelines and analytical formulations to deal with the practical implementation of negative capacitance circuits are provided.
\end{abstract}

Keywords: piezoelectric shunt, negative capacitance, vibration control, damping, smart structure

(Some figures may appear in colour only in the online journal)

\section{Introduction}

The use of piezoelectric actuators shunted with electric impedances in order to damp structural vibrations is an attractive approach because such lightweight control devices do not cause high load effects and require little or no power supply to control the structure. Such control methods can be classified as passive if the electric components used to practically build the impedance are passive (e.g. composed of a single resistance (resistive (R) shunt) or the series of a resistance and an inductance (resonant (RL) shunt)) [1], semipassive if the electronic circuit needs to be powered but is still theoretically equivalent to a passive one (in the case of a synthetic inductance, for instance) [2], or active. The most common example of an active circuit in the context of piezoelectric shunt is the negative capacitance (NC), practically realised with an operational amplifier (OP-AMP). The use of NCs enables the attenuation performance of the shunt to be increased [3, 4], but poses some issues relating to possible instabilities in the electro-mechanical system (EMS) because of its active nature.

Some aspects relating to the use of NCs to enhance the performances of shunted piezoelectric actuators have been addressed in the literature. Indeed, the use of circuits based on OP-AMPs to shunt piezoelectric actuators has been described in different pieces of work (e.g. [5]). Among the different circuits proposed, the NC was shown to be effective for vibration control. De Marneffe and Preumont [3] analysed the simplest layouts, named ideal circuits (IC) here, to build the NCs, and gave the stability criteria. In contrast, other authors 
have focused on more complex circuits, named real circuits (RC) here, because they are more reliable for practical implementation. The RCs cannot be considered as pure NCs (see section 5) but as complex negative impedances, while ICs can be seen as pure NCs. Behrens et al [4] considered an $\mathrm{RC}$ and showed its capability to provide broadband vibration attenuation when shunted to piezoelectric actuators. Park and Baz [6] shunted a pair of interdigital electrode piezoceramics with an NC to achieve broadband vibration control for a cantilevered beam. The works of Behrens et al and of Park and Baz differ in how to shunt the NC to the piezoelectric element (series and parallel respectively). Manzoni et al [7] analysed the stability of an RC in a series layout and Kodejška et al [8] used an RC in a series configuration in an adaptive circuit for the purpose of vibration isolation. Beck et al proposed an electrical model of an RC in a series layout in [9] and presented a procedure for improving the attenuation performance provided by an RC in a series configuration in [10].

An NC can be coupled to any passive (or semi-passive) shunt in order to increase its performance-especially the traditional R- and RL-shunts. The latter solution allows the attenuation provided by the pure resistive shunt to be improved a lot, despite losing its robustness [11]. The idea followed in this article is to use a negative capacitance to increase the attenuation performance of a pure resistive shunt while keeping its robustness. This article also addresses a tuning strategy to obtain multi-mode attenuation by means of a negative capacitance resistive shunt, whereas vibration attenuation is localised in a narrow frequency band around a given resonance for traditional R and RL shunts.

One of the purposes of the paper is to propose and analyse a new shunt impedance configuration based on two $\mathrm{NCs}$, in order to enhance the performance of traditional NC circuits coupled to a resistive shunt. In doing so, traditional layouts for NCs are reconsidered here. Indeed, although the use of an $\mathrm{NC}$ with a resistive shunt has already been described in the literature, no clear criteria for tuning the electric parameters and optimising shunt performance are available. Furthermore, different layouts can be used to practically build an NC circuit as mentioned previously, and some of them are seldom taken into account or analysed in the literature. Finally, no clear evaluation of the possible performances attainable with a negative capacitance are available. Therefore, for all the above-mentioned issues, the main targets of this paper are (i) to analyse most of the possible practical NC configurations, showing that a common analytical treatment can be used (see section 2); (ii) to propose a refined model for piezoelectric patch capacitance in order to derive accurate stability limits when NCs are used and to achieve a more accurate description of EMS behaviour (refer to sections 2 and 6); (iii) to propose a new layout based on the use of two $\mathrm{NCs}$ able to guarantee better vibration attenuation with respect to the traditional NC layouts (see section 3); (iv) to provide clear optimisation formulas to be employed in order to reach the highest possible attenuation for both mono-modal and broadband attenuation and (v) to derive analytical formulas which foresee the attenuation performance of the shunt,

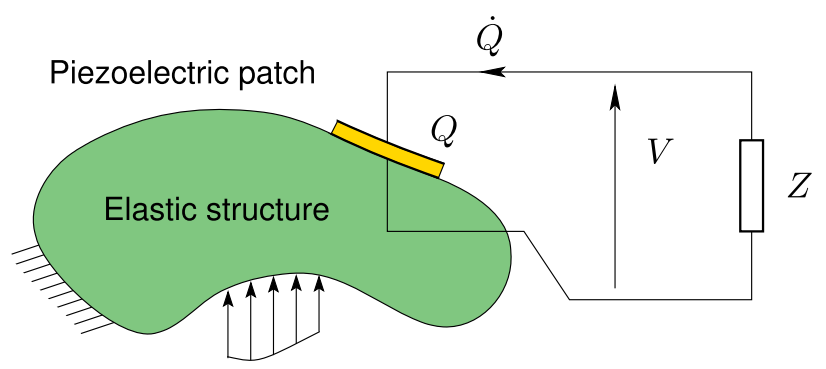

Ext. forcing

Figure 1. An arbitrary structure with a piezoelectric patch connected to a shunt impedance $Z$.

valid for any NC configuration (refer to section 4); finally, (vi) to study the behaviour of RCs and compare it to that of ICs, for practical implementations (see section 5). To conclude, all the aforementioned theoretical results are validated by experiments (refer to section 6).

\section{Model of the electro-mechanical system}

\subsection{Governing equations}

The model employed to describe the behaviour of the EMS is the one presented in the paper of Thomas et al [2], which was used by its authors to find the optimal tuning of $R$ and $R L$ impedances shunted to a piezoelectric actuator as well as the associated vibration attenuation performances. We consider an arbitrary elastic structure with one piezoelectric patch bonded to it and excited by an external force (named $F_{\text {ext }}$ ), shown in figure 1 . An arbitrary shunt impedance $Z$ is connected to the piezoelectric patch and $V$ is the voltage between the electrodes of the piezoelectric actuator, which is also the shunt terminal voltage. $Q$ is the electric charge in one of the electrodes, and considering the convention of the sign for $V$ in figure $1, Q$ is precisely the charge in the upper electrode. A reduced order model can be obtained by expressing the displacement $U(x, t)$ at any point $x$ and time $t$ in modal coordinates and considering the $N$ vibration eigenmodes ( $N$ being infinite in theory):

$$
U(x, t)=\sum_{i=1}^{N} \Phi_{i}(x) q_{i}(t)
$$

where $\Phi_{i}$ is the $i$ th eigenmode of the structure (normalised to the unit modal mass) and $q_{i}$ is the $i$ th modal coordinate. The modal coordinates $q_{i}(t)$ are the solutions to a problem of the form $[12,13]$ :

$$
\begin{gathered}
\ddot{q}_{i}+2 \xi_{i} \omega_{i} \dot{q}_{i}+\omega_{i}^{2} q_{i}-\chi_{i} V=F_{i} \quad \forall i \in 1 \ldots N \\
C_{\infty} V-Q+\sum_{i=1}^{N} \chi_{i} q_{i}=0
\end{gathered}
$$

In the above equations, $\omega_{i}$ denotes the $i$ th natural frequency of the EMS in short circuit (SC, i.e. with $Z=0$ ), $\xi_{i}$ is the $i$ th structural damping factor, $F_{i}$ is the harmonic modal forcing term, $C_{\infty}$ is the electrical capacitance of the piezoelectric patch (its physical meaning will be discussed in section 2.2) 
and $\chi_{i}$ is the modal coupling coefficient, which represents the energy transfer between the $i$ th mode shape and the piezoelectric patch. The values of $\chi_{i}$ depend on the electrical, geometrical and mechanical characteristics of the piezoelectric actuator and structure, and on the position of the actuator. These $\chi_{i}$ coefficients can be computed analytically [12] or by a finite element model [13], or estimated experimentally. The writing of equation (2a) relies on a chosen normalisation of the mode shapes to have unit modal masses. The unit of $\Phi_{i}$ is therefore $\mathrm{kg}^{-1 / 2}$, the unit of $q_{i}$ is $\mathrm{m} \cdot \mathrm{kg}^{1 / 2}$, and the one of $F_{i}$ is $\mathrm{N} \cdot \mathrm{kg}^{-1 / 2}$, so that the unit of $\chi_{i}$ is either $\mathrm{N} \cdot \mathrm{V}^{-1} \cdot \mathrm{kg}^{-1 / 2}$ or $\mathrm{C} \cdot \mathrm{m}^{-1} \cdot \mathrm{kg}^{-1 / 2}$, depending on whether the direct or converse piezoelectric effects are considered. Note that $\left(\Phi_{i}, \omega_{i}\right)$ are the eigenmodes of the EMS with the piezoelectric patch in short circuit (SC) (with $V=0$ ) and that $C_{\infty}$ is the patch capacitance of the blocked structure (with $\left.U(x, t)=0 \forall x \Rightarrow q_{i}=0 \forall i\right)$. As for a rectangular piezoelectric patch with a constant thickness $h$, the patch capacitance of the blocked structure can be computed theoretically by [13]:

$$
C_{\infty}=\bar{\epsilon}_{33} S / h
$$

where $S$ is the area of the electrodes and $\bar{\epsilon}_{33}$ is the modified dielectric permittivity at constant strain, in the patch transverse direction, of the piezoelectric material.

The EMS is thus described by $N$ modal equations (equation (2a)), corresponding to the balance law of mechanical forces, which describe the equations of motion of the EMS. These equation (2a) are coupled by the term $\chi_{i} V$ to equation ( $2 b$ ), which represents the electrical behaviour of the system. Equation (2b) is related to the balance of electric charges on the piezoelectric patch electrodes.

\subsection{Model of the electrical capacitance and static correction}

The piezoelectric actuator and the structure are physically linked, and thus the dynamics of the structure is affected by the electrical behaviour of the EMS through the coupling coefficients $\chi_{i}$. On the other hand, the electrical behaviour of the system is in turn affected by the dynamics of the structure. Indeed, the expression of an equivalent capacitance can be derived from equations (2a), (2b). The expression of such an equivalent capacitance is frequency- dependent and can be obtained solving equation (2a) with respect to $q_{i}$ in the frequency domain (with $F_{i}=0$ ) and substituting the result in equation (2b). If all variables oscillate at a frequency $\Omega$, the equivalent capacitance of the EMS is:

$$
C(\Omega)=\frac{\underline{Q}(\Omega)}{\underline{V}(\Omega)}=C_{\infty}+\sum_{i=1}^{N} \frac{\chi_{i}^{2}}{\omega_{i}^{2}-\Omega^{2}+2 j \xi_{i} \omega_{i} \Omega}
$$

where $j$ is the complex imaginary unit and $Q$ and $\underline{V}$ are the complex amplitudes of $Q$ and $V$.

The trend of $C(\Omega)=Q / \underline{V}$ for a generic system is depicted in figure 2(a). It shows that the value of $C(\Omega)$ decreases after each resonance of the system, because of the second term of equation (4). As for the high frequency range (at frequencies above the highest natural frequency of the EMS), $C(\Omega) \simeq C_{\infty}$. As a consequence, the capacitance $C_{\infty}$ of the blocked structure, which naturally appears in the model (2a), (2b) (see equation (3)), is also the value of the capacitance of the piezoelectric patch at high frequency. In the low frequency range, below the first natural frequency of the EMS, $C(\Omega) \simeq C_{0}$ with:

$$
C_{0}=C_{\infty}+\sum_{i=1}^{N} \frac{\chi_{i}^{2}}{\omega_{i}^{2}} .
$$

If the natural frequencies of the modes are well separated, a single-degree-of-freedom approximation can be carried out to describe the behaviour of the EMS for $\Omega \simeq \omega_{i}$. Therefore, the second term of equation (4) can be approximated by keeping only the contribution of the $i$ th mode in the sum, so that:

$$
\begin{aligned}
& \Omega \simeq \omega_{i} \Rightarrow C(\Omega) \simeq C_{\infty}+\frac{\chi_{i}^{2}}{\omega_{i}^{2}-\Omega^{2}+2 j \xi_{i} \omega_{i} \Omega}, \\
& \forall i=1 \ldots N
\end{aligned}
$$

This simple one-degree-of-freedom (dof) model is not accurate in describing the behaviour of the capacitance around the mode considered (see figure 2(b)). It can be corrected by adding the low and high frequency contributions of all the modes which are out of the frequency band of interest. At the frequencies $\Omega$ around $\omega_{i}$, the contribution of all the modes $n$ at a lower frequency $\left(\omega_{n} \ll \omega_{i}\right)$ is zero because the corresponding term in the sum of equation (4) tends to zero for $\Omega \gg \omega_{n}$. All the modes $n$ of higher frequency $\left(\omega_{n} \gg \omega_{i}\right)$ contribute with a static component $\chi_{n}^{2} / \omega_{n}^{2}$, since in this case $\Omega \ll \omega_{n}$. As a consequence, a refined one-degree-of-freedom model for $C(\Omega)$ is:

$$
\begin{aligned}
& \Omega \simeq \omega_{i} \Rightarrow C(\Omega) \simeq C_{\mathrm{p} i}+\frac{\chi_{i}^{2}}{\omega_{i}^{2}-\Omega^{2}+2 j \xi_{i} \omega_{i} \Omega}, \\
& \forall i=1 \ldots N
\end{aligned}
$$

with

$$
C_{\mathrm{p} i}=C_{\infty}+\sum_{n=i+1}^{N} \frac{\chi_{n}^{2}}{\omega_{n}^{2}}, \quad \forall i=1 \ldots N
$$

In other words, the capacitance value $C_{\mathrm{p} i}$ at a given frequency does not depend on the modes of the structure at lower frequency, but just on those at higher frequency.

As a consequence, a refined one-degree-of-freedom model of the EMS, valid in a frequency band around a given resonance $\left(\Omega \simeq \omega_{i}\right.$ ), is obtained by keeping only the $i$ th mode in the expansion $\left(q_{n}=0, \forall n \neq i\right)$. Therefore, equation (1) is replaced by $U(x, t)=\Phi_{i}(x) q_{i}(t)$, equation (2a) is kept and equation (2b) is replaced by:

$$
C_{\mathrm{p} i} V-Q+\chi_{i} q_{i}=0,
$$

where the static correction due to the higher modes is included in the term $C_{\mathrm{p} i}$.

Figure 2(b) shows how it is possible to improve the accuracy of the model of the capacitance using equation (7) in place of equation (6) in the frequency band around the mode taken into account. Figure 2(b) shows that the use of $C_{\mathrm{p} i}$ 


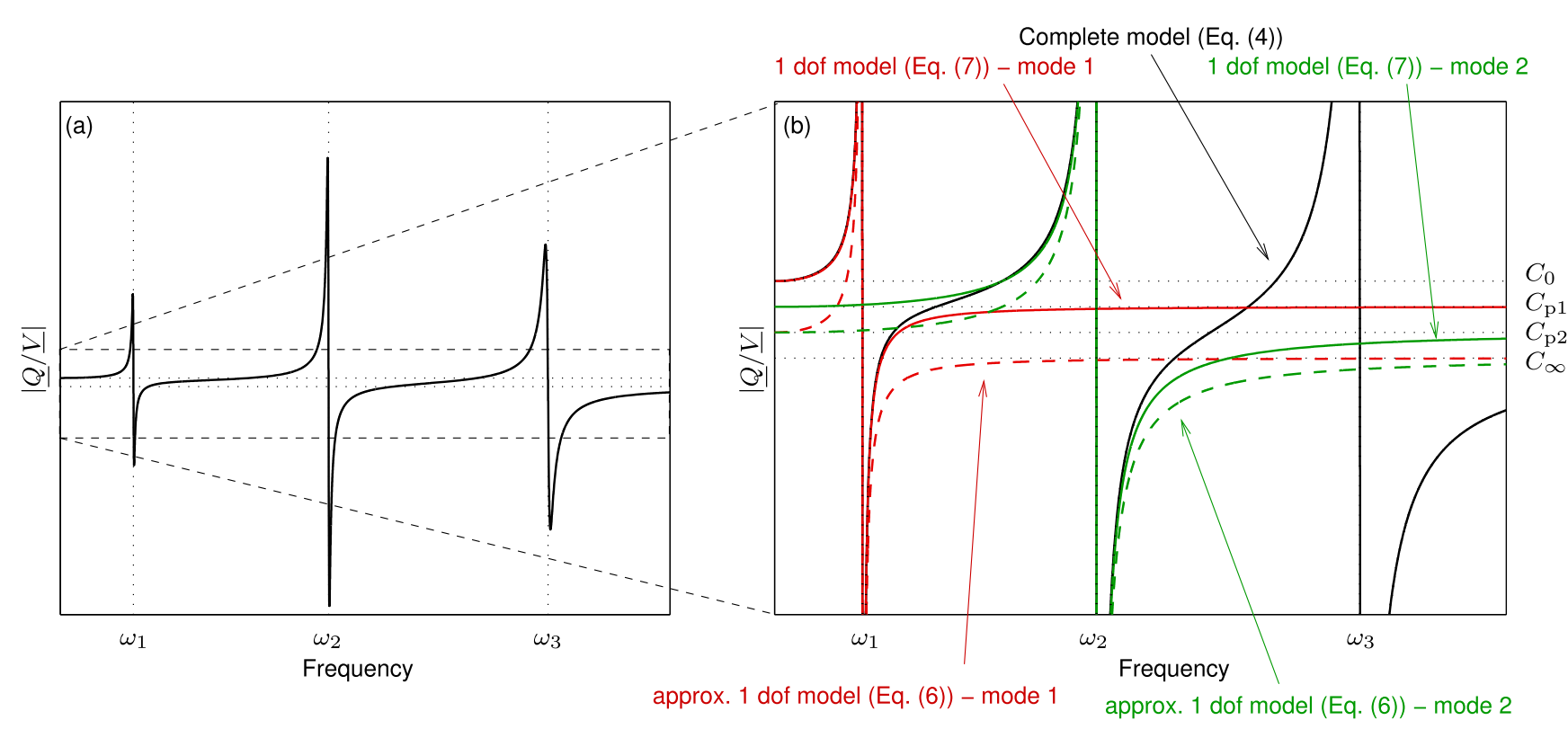

Figure 2. The trend of $C(\omega)=|Q / \underline{V}|$ for a generic, undamped three-degrees-of-freedom system. (a) The complete model of equation (4); (b) zoom showing the complete model (black) of equation (4) and the 1 dof models of equation (6) (dashed lines) and (7) (solid lines) tuned to mode 1 (red) and mode 2 (green). Note that the continuous lines relating to equations (4) and (7) (red line, first mode) are fully superimposed before $\omega_{1}$.

(equation (7)) leads to a 1 dof model which almost merges with the complete model of equation (4) around a given resonance; this is far from being the case for the classical simple model of equation (6) with $C_{\infty}$. Figure 2(b) also shows that $C(\Omega)$ assumes the value $C_{\mathrm{p} i}$ almost midway between the resonance frequencies of mode $i$ and $i+1$.

\subsection{Coupling factor}

In order to introduce the electromechanical coupling factor, we apply the following changes of variables:

$$
\bar{V}=V \sqrt{C_{\mathrm{p} i}}, \quad \bar{Q}=\frac{Q}{\sqrt{C_{\mathrm{p} i}}}
$$

Substituting equation (10) into equations (2a) and (9), a normalised formulation to describe the EMS behaviour is obtained:

$$
\begin{gathered}
\ddot{q}_{i}+2 \xi_{i} \omega_{i} \dot{q}_{i}+\omega_{i}^{2} q_{i}-\omega_{i} k_{i} \bar{V}=F_{i}, \\
\ddot{q}_{i}+2 \xi_{i} \omega_{i} \dot{q}_{i}+\hat{\omega}_{i}^{2} q_{i}-\omega_{i} k_{i} \bar{Q}=F_{i}, \\
\bar{V}-\bar{Q}+\omega_{i} k_{i} q_{i}=0,
\end{gathered}
$$

where:

$$
k_{i}=\frac{\chi_{i}}{\omega_{i} \sqrt{C_{\mathrm{p} i}}}, \quad \text { and } \quad \hat{\omega}_{i}=\omega_{i} \sqrt{1+k_{i}^{2}} .
$$

Equations (11a) and (11b) are equivalent, both expressing the converse piezoelectric effect, as a function of either $\bar{V}$ or $\bar{Q}$. Equation (11b) is obtained by substituting $\bar{V}$ in favour of $\bar{Q}$ in equation (11a) using (11c). The non-dimensional parameter $k_{i}$ is defined as the modal electromechanical coupling factor (MEMCF). The short- (SC) and open-circuit (OC) natural frequencies of the EMS are $\omega_{i}$ and $\hat{\omega}_{i}$ (respectively associated with zero $(Z=0)$ and infinite $(Z=\infty)$ shunt impedances, also obtained with $\bar{V}=0$ and $\bar{Q}=0$ respectively). Equation (12) shows that the MEMCF $k_{i}$ is close to the $i$ th effective coupling factor:

$$
k_{\mathrm{eff}}=\sqrt{\frac{\hat{\omega}_{i}^{2}-\omega_{i}^{2}}{\omega_{i}^{2}}}=\left|k_{i}\right|
$$

As suggested in $[3,14]$, the SC natural frequencies $\omega_{i}$ are the poles of $C(\Omega)$ (equation (4)) and the OC natural frequencies $\hat{\omega}_{i}$ are its zeros. Since $k_{i}$ is dimensionless, another unit for $\chi_{i}$ is $\mathrm{F}^{1 / 2} \cdot \mathrm{s}^{-1}$.

By considering the definition of $k_{i}$ in equation (12) together with equation (8), it can be derived that the values of $C_{\mathrm{p} i}$ for two consecutive modes in the spectrum are linked by:

$$
C_{\mathrm{p} i}=C_{\mathrm{pi}+1}\left(1+k_{i+1}^{2}\right)
$$

Consequently, the value of the capacitance at the null frequency can be derived as a function of all the modes of the structure:

$$
C_{0}=C_{\infty}+\sum_{i=1}^{N} C_{\mathrm{p} i} k_{i}^{2}
$$

The following relations for the different capacitance values hold:

$$
C_{0}>C_{\mathrm{p} 1}>\ldots>C_{\mathrm{p} i}>\ldots>C_{\infty}
$$

These results are illustrated in figure 2(b): the capacitance, out of the resonance zones, changes by a factor almost equal to $C_{\mathrm{p} i} k_{i}^{2}$ at each resonance crossing. 

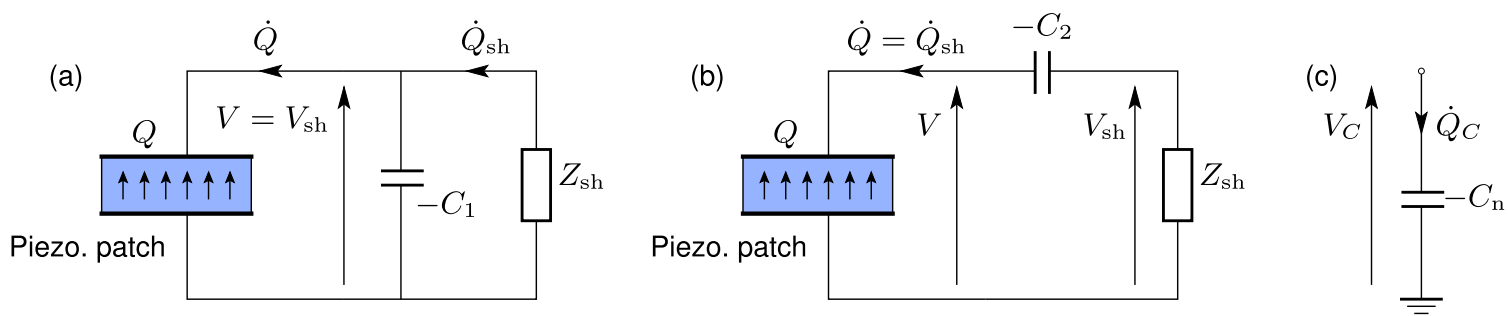

Figure 3. Shunt with the addition of an NC: (a) parallel configuration; (b) series configuration. (c) Definition of an NC.

Table 1. Parameters of the EMS enhanced by a single NC in a parallel and series configuration and by two NCs for the series + parallel (SP) configuration.

\begin{tabular}{|c|c|c|c|c|c|}
\hline & & R-shunt & Parallel config. & Series config. & SP config. \\
\hline $\mathrm{SC}$ freq. & $\omega_{i}^{\mathrm{sc}}$ & $\omega_{i}$ & $\omega_{i}$ & $\sqrt{\omega_{i}^{2}-\frac{\chi_{i}^{2}}{C_{2}-C_{\mathrm{p} i}}}$ & $\sqrt{\omega_{i}^{2}-\frac{\chi_{i}^{2}}{C_{1}+C_{2}-C_{\mathrm{p} i}}}$ \\
\hline OC freq. & $\omega_{i}^{\mathrm{oc}}$ & $\hat{\omega}_{i}=\sqrt{\omega_{i}^{2}+\frac{\chi_{i}^{2}}{C_{\mathrm{p} i}}}$ & $\sqrt{\omega_{i}^{2}+\frac{\chi_{i}^{2}}{C_{\mathrm{p} i}-C_{1}}}$ & $\hat{\omega}_{i}=\sqrt{\omega_{i}^{2}+\frac{\chi_{i}^{2}}{C_{\mathrm{p} i}}}$ & $\sqrt{\omega_{i}^{2}+\frac{\chi_{i}^{2}}{C_{\mathrm{p} i}-C_{1}}}$ \\
\hline EMEMCF & $\tilde{k}_{i}$ & $k_{i}$ & $\frac{k_{i}}{\sqrt{1-\frac{C_{1}}{C_{\mathrm{p} i}}}}$ & $\frac{k_{i}}{\sqrt{1-\frac{C_{\mathrm{p} i}}{C_{2}}}}$ & $\frac{k_{i}}{\sqrt{\left(1-\frac{C_{1}}{C_{\mathrm{p} i}}\right)\left(1+\frac{C_{1}}{C_{2}}-\frac{C_{\mathrm{p} i}}{C_{2}}\right)}}$ \\
\hline Elec. time const. & $\tau_{e}$ & $R_{\mathrm{sh}} C_{\mathrm{p} i}$ & $R_{\mathrm{sh}}\left(C_{\mathrm{p} i}-C_{1}\right)$ & $R_{\mathrm{sh}} \frac{C_{\mathrm{p} i} C_{2}}{C_{2}-C_{\mathrm{p} i}}$ & $R_{\mathrm{sh}} \frac{\left(C_{\mathrm{p} i}-C_{1}\right) C_{2}}{C_{1}+C_{2}-C_{\mathrm{p} i}}$ \\
\hline
\end{tabular}

\subsection{EMS with negative capacitance}

Once the model of the EMS (section 2.3) has been described, we can consider the case in which an NC is connected to the piezoelectric actuator, in addition to the shunt impedance, now denoted by $Z_{\mathrm{sh}}$. There are two classical configurations for the NC: a parallel configuration (figure 3(a)) or a series configuration (figure 3(b)). Both layouts are characterised by the presence of an additional negative capacitance $-C_{1}$ or $-C_{2}$. This element is theoretically defined by the following relation between its terminal voltage $V_{c}$ and its charge $Q_{c}$ (see figure 3(c)):

$$
V_{c}=-\frac{Q_{c}}{C_{n}}
$$

Since NCs do not exist in nature, they are built in practice by means of a circuit including an OP-AMP [15]. Such a circuit will be discussed in detail in section 5 .

In the following, we focus our attention on the response of the EMS due to the $i$ th vibration mode $\left(q_{n}=0 \forall n \neq i\right)$, using the model of equation (9) with the static correction. As for the parallel configuration (figure 3(a)), the charge $Q$ is the sum of the charges in the negative capacitance $-C_{1}$ branch and the shunt impedance $Z_{\mathrm{sh}}$ branch, so that equation (2b) is replaced by:

$$
\left(C_{\mathrm{p} i}-C_{1}\right) V-Q_{\mathrm{sh}}+\chi_{i} q_{i}=0
$$

As for the series configuration, the voltage $V$ is the sum of the voltages at the terminals of the negative capacitance $-C_{2}$ and the shunt impedance $Z_{\mathrm{sh}}$, so that equation ( $2 \mathrm{~b}$ ) is replaced by:

$$
V_{\mathrm{sh}}-\left(\frac{1}{C_{\mathrm{p} i}}-\frac{1}{C_{2}}\right) Q+\frac{\chi_{i}}{C_{\mathrm{p} i}} q_{i}=0
$$

In the above equations, $V_{\mathrm{sh}}$ and $Q_{\mathrm{sh}}$ denote the terminal voltage across the shunt impedance and the charge that flows into the shunt impedance branch, respectively.

It is now convenient to define the following equivalent capacitances:

$$
C_{\text {eqp }}=C_{\mathrm{p} i}-C_{1}, \quad C_{\mathrm{eqs}}=\frac{C_{\mathrm{p} i} C_{2}}{C_{2}-C_{\mathrm{p} i}} .
$$

These are related to the configuration with $-C_{1}$ in parallel to the piezoelectric patch and $-C_{2}$ in series with the piezoelectric patch respectively. By applying a change of variables analogous to that in equation (10):

$$
\bar{V}_{\mathrm{sh}}=V_{\mathrm{sh}} \sqrt{C_{\mathrm{eq}}} \quad \bar{Q}_{\mathrm{sh}}=\frac{Q_{\mathrm{sh}}}{\sqrt{C_{\mathrm{eq}}}}
$$

with $C_{\text {eq }}=C_{\text {eqp }}$ or $C_{\text {eq }}=C_{\text {eqs }}$, the initial problem (11a)-(11c) can be rewritten in the following form:

$$
\begin{gathered}
\ddot{q}_{i}+2 \xi_{i} \omega_{i} \dot{q}_{i}+\left(\omega_{i}^{\mathrm{sc}}\right)^{2} q_{i}-\omega_{i} \tilde{k}_{i} \bar{V}_{\mathrm{sh}}=F_{i}, \\
\ddot{q}_{i}+2 \xi_{i} \omega_{i} \dot{q}_{i}+\left(\omega_{i}^{\mathrm{oc}}\right)^{2} q_{i}-\omega_{i} \tilde{k}_{i} \bar{Q}_{\mathrm{sh}}=F_{i}, \\
\bar{V}_{\mathrm{sh}}-\bar{Q}_{\mathrm{sh}}+\omega_{i} \tilde{k}_{i} q_{i}=0
\end{gathered}
$$

The above set of equations defines the dynamics of the EMS viewed from the shunt impedance $Z_{\text {sh }}$ since the electrical unknowns are now $\bar{V}_{\mathrm{sh}}$ and $\bar{Q}_{\mathrm{sh}}$. The following parameters have been defined: $\omega_{i}^{\text {sc }}$ and $\omega_{i}^{\text {oc }}$ are respectively the natural frequency of the EMS with the shunt $Z_{\text {sh }}$ short-circuited $\left(V_{\mathrm{sh}}=0 \Leftrightarrow Z_{\mathrm{sh}}=0\right)$ and in open-circuit $\left(Q_{\mathrm{sh}}=0 \Leftrightarrow Z_{\mathrm{sh}}=\right.$ $\infty)$. Their values depend on the values of the negative capacitances $-C_{1}$ and $-C_{2}$. The above model characterises the behaviour of the EMS grouped with the negative capacitances $-C_{1}$ or $-C_{2}$, whose apparent capacitance is $C_{\text {eqp }}$ or $C_{\text {eqs }}$, instead of $C_{\mathrm{p} i}$. A new coupling factor naturally appears in the equations: the enhanced modal electromechanical coupling 


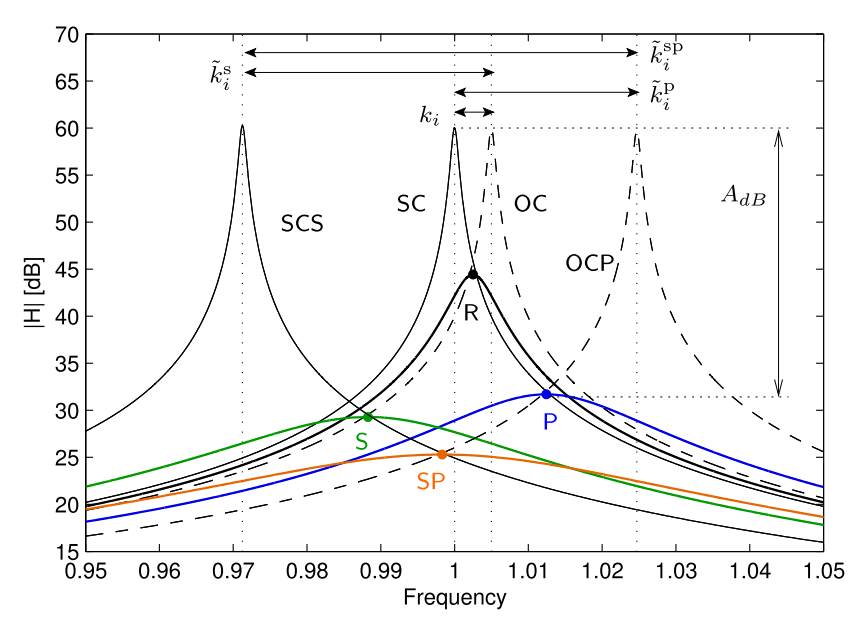

Figure 4. The FRFs (equation (26)) of a 1 dof EMS associated with several resistive shunt circuits and several tunings of $R_{\mathrm{sh}}\left(\tau_{e}\right)$. SC and OC: short- and open-circuit response without a NC; OCP: opencircuit response with a negative capacitance in parallel configuration; SCS: short-circuit response with a negative capacitance in series configuration; R (R-shunt), P (parallel), S (series) and SP (series +parallel): response of the EMS with the optimal value of $R_{\mathrm{sh}}\left(\tau_{e}\right)$ for each NC layout.

factor (EMEMCF) $\tilde{k}_{i}$, that can be written as:

$$
\left|\tilde{k}_{i}\right|=\sqrt{\frac{\left(\omega_{i}^{\mathrm{oc}}\right)^{2}-\left(\omega_{i}^{\mathrm{sc}}\right)^{2}}{\omega_{i}^{2}}},
$$

similar to that of equation (13). One can notice that $\tilde{k}_{i}$ is not exactly the traditional effective coupling factor since now the denominator is $\omega_{i}$ and not $\omega_{i}^{\text {sc }}$. These parameters, which take different values in the parallel and series configurations, are gathered in table 1. Their dependence upon the negative capacitances $C_{1}$ or $C_{2}$ will be discussed in detail in sections 2.6 and 2.7 .

It is worth highlighting that equations (22a)-(22c), which describe the EMS with an NC (series or parallel), are valid even for a simple shunt (without NC). Indeed, it is sufficient to substitute in the model the original $\omega_{i}$ and $\hat{\omega}_{i}$ in place of $\omega_{i}^{\text {sc }}$ and $\omega_{i}^{\text {oc }}$ and $k_{i}$ in place of $\tilde{k}_{i}$. Therefore, the model described by equations (22a)-(22c) can be considered general and valid for simple and NC shunts.

\subsection{Resistive shunt}

We now consider a resistance as shunt impedance (i.e. $Z_{\mathrm{sh}}=R_{\mathrm{sh}}$ in figure 3 ). This leads to $V_{\mathrm{sh}}=-R_{\mathrm{sh}} \dot{Q}_{\mathrm{sh}}$, so that equation $(22 \mathrm{c})$ becomes:

$$
\tau_{e} \dot{\bar{Q}}_{\mathrm{sh}}+\bar{Q}_{\mathrm{sh}}-\omega_{i} \tilde{k}_{i} q_{i}=0 .
$$

With the electrical time constant

$$
\tau_{e}=R_{\mathrm{sh}} C_{\mathrm{eq}}
$$

One has to remark that $\tau_{e}$ also depends on the considered $i$ th mode because $C_{\text {eq }}$ depends on $C_{\mathrm{p} i}$.

Consequently, relying on equations (22b) and (24), the frequency response function (FRF) $H_{i}(\Omega)$ between a harmonic modal force $F_{i}$ of frequency $\Omega$ and $q_{i}$ is:

$$
\begin{aligned}
& H_{i}(\Omega)=\frac{q_{i}}{F_{i}}= \\
& \frac{1+j \tau_{e} \Omega}{\left(\omega_{i}^{\mathrm{sc}}\right)^{2}-\left(1+2 \xi_{i} \omega_{i} \tau_{e}\right) \Omega^{2}+j \Omega\left[\tau_{e}\left(\omega_{i}^{\mathrm{oc}}\right)^{2}+2 \xi_{i} \omega_{i}-\tau_{e} \Omega^{2}\right]}
\end{aligned}
$$

\subsection{Stability conditions for ideal series and parallel circuits}

The introduction of active elements such as OP-AMP makes the stability analysis of the EMS compulsory. One possible approach is to apply the Routh-Hurwitz criterion [16] to the controlled FRF. This guarantees having all poles with negative real parts [17]. If we consider the FRF of a single-degreeof-freedom EMS defined in equation (26), the stability condition for the parallel configuration is found to be:

$$
C_{1}<C_{\mathrm{p} i}
$$

and for the series configuration:

$$
C_{2}>C_{\mathrm{p} i}\left(1+k_{i}^{2}\right)=C_{\mathrm{p} i-1}
$$

These conditions are obtained by considering in the FRF of equation (26) the expressions of $\omega_{i}^{\text {oc }}$ and $\omega_{i}^{\text {sc }}$ defined in table 1 .

Nonetheless, when stability issues are faced, it is mandatory to model the whole system without neglecting any mode. Indeed, if the model of the EMS takes into account just some of the modes, spillover problems can occur. This means that the stability of the EMS must be studied starting from the complete model of equation (2a) together with equations (18) and (19) without applying the truncation to a single-degreeof-freedom system.

The stability conditions for the whole EMS are related to the strictest conditions among all the modes defined by equations (27) and (28). As for the parallel configuration, the strictest condition is the one related to the highest mode of the EMS:

$$
C_{1}<C_{\infty}
$$

whereas, the strictest condition for the series layout is given by the lowest (i.e. the first) mode of the EMS:

$$
C_{2}>C_{0}
$$

These conditions are in accordance with those proposed by de Marneffe and Preumont [3]. Intuitively, the stability limits can also be related to the effects of the $\mathrm{NC}$ on the values of $\omega_{i}^{\text {oc }}$ and $\omega_{i}^{\text {sc }}$. In the parallel configuration, if $C_{1} \rightarrow C_{\infty}, \omega_{i}^{\text {oc }}$ of the highest mode tends to $+\infty$ (see table 1 ). Thus, the destabilisation process in this case comes from a high-frequency mode whose open-circuit frequency reaches $+\infty$. In contrast, for the series configuration, if $C_{2} \rightarrow C_{0}, \omega_{i}^{\mathrm{sc}}$ of the first mode tends to zero. Thus, the destabilisation comes from a low-frequency mode whose short-circuit frequency reaches 0 . 


\subsection{Effect of negative capacitance}

The MEMCF without any NC for mode $i$ is $k_{i}$, defined by equation (12). When we consider a negative capacitance in parallel or series configuration, we have defined an enhanced coupling factor $\tilde{k}_{i}$, which is the coupling factor of the EMS viewed from the shunt impedance $Z_{\mathrm{sh}}$, and thus including the negative capacitance. Table 1 shows the values of $\tilde{k}_{i}$ (see equation (23)), which are always higher than $k_{i}$ if the stability conditions of section 2.6 are fulfilled. The main effect of the negative capacitance is to artificially ${ }^{4}$ increase the MEMCF by changing the capacitance of the piezoelectric patch viewed from the shunt (the equivalent capacitance for series and parallel configurations, given by equations (20)). According to Thomas et al [2], the higher the MEMCF is, the better the vibration attenuation provided by the resistive shunt is. Thus, the addition of the $\mathrm{NC}$ allows the attenuation performance provided by the shunt control to be artificially improved.

The increase in the MEMCF can also be viewed as an increase in the distance between the open- and short-circuit eigenfrequencies (see table 1), as shown in figure 4 . As for the parallel configuration, the short-circuit eigenfrequencies are not changed by the presence of the $\mathrm{NC}$, whereas the opencircuit eigenfrequencies are shifted towards higher frequency values. Note that the closer $C_{1}$ is to the stability limit, the higher $\omega_{i}^{\text {oc }}$ and $\tilde{k}_{i}$ are. As for the series configuration, the open-circuit eigenfrequencies are not changed by the presence of the NC, but the short-circuit eigenfrequencies are shifted towards lower frequency values. The closer $C_{2}$ is to the stability limit, the lower $\omega_{i}^{\text {sc }}$ is and the higher $\tilde{k}_{i}$ is.

The addition of the NC makes it possible to increase the MEMCF by shifting either the open- or short-circuit eigenfrequency, depending on the way the $\mathrm{NC}$ is connected to the piezoelectric patch and the resistance $R_{\mathrm{sh}}$ (i.e. in parallel or series). The next section proposes a new shunt circuit layout with a couple of NCs and a resistance. The use of two NCs together allows both the open- and short-circuit eigenfrequencies to be shifted at the same time. Thus, the increase in the MEMCF is higher than that achievable by using parallel and series configurations. A detailed comparison of the enhancement of the MEMCF provided by the three configurations (i.e. series, parallel and the new one) will be given in the next section.

\section{A new electrical circuit layout with negative capacitances}

The previous sections showed that the use of an NC artificially increases the MEMCF. The idea behind the new circuit proposed herein is to increase the open-circuit eigenfrequencies and to decrease the short-circuit eigenfrequencies at the same time by using two NCs: one connected in parallel $\left(-C_{1}\right)$ and the other in series $\left(-C_{2}\right)$, as in evidence in figure 5.

4 We say artificially because the MEMCF is an intrinsic property of the EMS that depends on the piezoelectric material and on the geometry of the system.

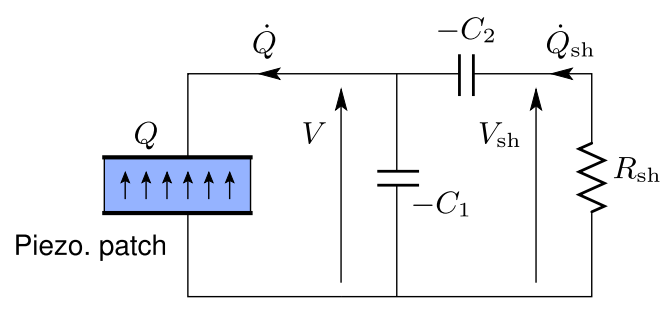

Figure 5. The new proposed circuit with two NCs.

Therefore, this new configuration is named the series + parallel (SP) configuration.

\subsection{Analytical model}

Relying on figure 5 and following the same approach used in section 2.4 , equation ( $2 \mathrm{~b})$ is replaced by:

$$
\frac{\left(C_{\infty}-C_{1}\right) C_{2}}{\left(C_{1}+C_{2}-C_{\infty}\right)} V_{\mathrm{sh}}-Q_{\mathrm{sh}}+\sum_{i=1}^{N} \frac{\chi_{i} C_{2}}{C_{1}+C_{2}-C_{\infty}} q_{i}=0
$$

If this model is restricted to the $i$ th vibration mode $\left(q_{n}=0 \forall n \neq i\right)$, using the model proposed in equation (9) with the static correction, one defines the following equivalent capacitance:

$$
C_{\text {eqsp }}=\frac{\left(C_{\mathrm{p} i}-C_{1}\right) C_{2}}{C_{1}+C_{2}-C_{\mathrm{p} i}} .
$$

for the network made up by the three capacitances $\left(C_{\mathrm{p} i},-C_{1}\right.$ and $-C_{2}$ ).

Then, using the change of variable of equation (21) with $C_{\text {eq }}=C_{\text {eqsp }}$, the model of equations (22a), (22b), (22c) is obtained again with the following short- and open-circuit eigenfrequencies:

$$
\begin{gathered}
\omega_{i}^{\mathrm{sc}}=\sqrt{\omega_{i}^{2}-\frac{\chi_{i}^{2}}{C_{1}+C_{2}-C_{\mathrm{p} i}}} \\
\omega_{i}^{\mathrm{oc}}=\sqrt{\omega_{i}^{2}+\frac{\chi_{i}^{2}}{C_{\mathrm{p} i}-C_{1}}}
\end{gathered}
$$

and with $\tau_{e}=R_{\mathrm{sh}} C_{\text {eqsp. }}$.

As expected, the use of two NCs in the shunt circuit allows us to decrease the short-circuit eigenfrequencies and to increase the open-circuit ones at the same time, thus leading to an enhanced MEMCF $\tilde{k}$ :

$$
\tilde{k}_{i}=\frac{k_{i}}{\sqrt{\left(1-\frac{C_{1}}{C_{\mathrm{p} i}}\right)\left(1+\frac{C_{1}}{C_{2}}-\frac{C_{\mathrm{p} i}}{C_{2}}\right)}}
$$

This is illustrated in figure 4 .

The analytical formulation of the FRF of the SP configuration described here is exactly the same as that of the series and parallel configurations (and of the simple R-shunt, see section 2.4 and [2]), defined by equation (26), where the values of $\omega_{i}^{\mathrm{sc}}, \omega_{i}^{\mathrm{oc}}$ and $\tau_{e}$ to be considered depend on the NC layout used. The parameters involved in the definition of $H_{i}$ 


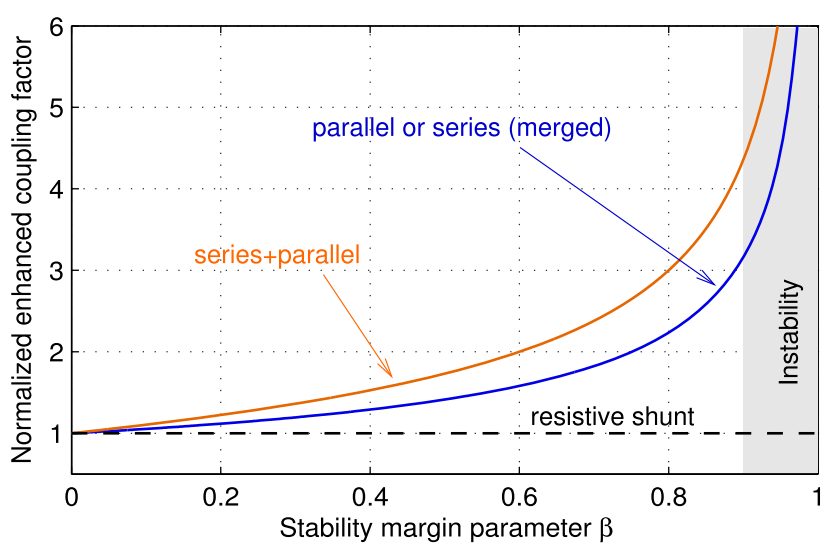

Figure 6 . The trend of the EMEMCF $\tilde{k}_{i}$, normalised by $k_{i}\left(\tilde{k}_{i} / k_{i}\right)$, as a function of $\beta$, for the three NC configurations (parallel, series and series+parallel), with $\beta=\beta_{\mathrm{p}}=\beta_{\mathrm{s}}=\beta_{\mathrm{sp} 1}=\beta_{\mathrm{sp} 2}$. The grey region shows an example of the region for which the system is unstable, for $\beta$ above 0.9 in this particular case.

are gathered in table 1 for the parallel, series and SP configurations.

\subsection{Stability conditions for the ideal SP configuration}

The stability condition for SP can be deduced by those of section 2.6 or, equivalently, by applying the Routh-Hurwitz criterion to the denominator of equation (26). The most restrictive conditions relating to the first and last modes (as explained in section 2.6) are:

$$
C_{1}<C_{\infty} \quad \text { and } \quad C_{1}+C_{2}>C_{0}
$$

In this case we found two stability conditions to be satisfied at the same time. Indeed, the open-circuit eigenfrequency of the highest mode must not shift to $+\infty$ and the short-circuit eigenfrequency of the first mode must not reach the null frequency.

\subsection{Effect on the MEMCF}

The new circuit proposed herein is able to increase the MEMCF more than the series and parallel configurations, as explained in the previous sections. A straightforward approach to having a quantitative understanding of the benefits provided by the SP configuration is possible by considering a single-degree-of-freedom system. We define four indexes able to describe how much the negative capacitance, related to each possible configuration, is far from $C_{\mathrm{p} i}: \beta_{1}$ for the parallel, $\beta_{2}$ for the series, and $\beta_{\mathrm{sp} 1}$ and $\beta_{\mathrm{sp} 2}$ for the SP configuration. Their expressions are:

$$
\begin{aligned}
\beta_{1}=\frac{C_{1}}{C_{\mathrm{p} i}}, & \beta_{2}=\frac{C_{\mathrm{p} i}}{C_{2}}, \\
\beta_{\mathrm{sp} 1}=\frac{C_{1}}{C_{\mathrm{p} i}}, & \beta_{\mathrm{sp} 2}=\frac{C_{\mathrm{p} i}}{C_{1}+C_{2}}
\end{aligned}
$$

With these definitions, null $\beta$ values correspond to a situation where no NCs are added to the circuit $\left(C_{1}=0\right.$ and
$\left.C_{2}=+\infty\right)$. In contrast, $\beta$ coefficients equal to one mean that the values of the NCs are beyond but close to the instability condition.

Table 2 gathers the values of $\omega_{i}^{\mathrm{sc}}, \omega_{i}^{\mathrm{oc}}, \tilde{k}_{i}$ and $\tau_{e}$ as a function of $\beta$, obtained with those of table 1. Figure 6 shows $\tilde{k}_{i} / k_{i}$ (i.e. normalised enhanced coupling factor) as a function of $\beta$ (achieved with the closed form expressions of the third row of table 2) and enables the benefit of the negative capacitance to be interpreted in terms of MEMCF enhancement. The two single capacitance configurations (series and parallel) give the same coupling factor enhancement for a given value of the $\beta$ index. Moreover, for any value of $\beta$, the SP configuration always displays higher $\tilde{k}_{i}$ values than a single negative capacitance in series or in parallel.

Since $\tilde{k}_{i}$ tends to $+\infty$ when $\beta$ approaches 1 , one might think it possible to design a negative capacitance circuit with infinite performance. However, the performances are limited by the stability limit (see equations (36), (30) and (29)), which are discussed here as a function of the $\beta$ indexes. The value of $\beta$, defined above, depends on $C_{\mathrm{p} i}$ and thus only on the $i$ th mode considered in the corresponding 1 dof model. Actually, the value(s) of $\beta$ for which the system becomes unstable depend on $C_{0}$ and $C_{\infty}$ (see sections 2.6 and 3.2):

$$
\beta_{1}^{\text {inst }}=\beta_{\mathrm{sp} 1}^{\text {inst }}=\frac{C_{\infty}}{C_{\mathrm{p} i}}, \quad \beta_{2}^{\text {inst }}=\beta_{\mathrm{sp} 2}^{\text {inst }}=\frac{C_{\mathrm{p} i}}{C_{0}},
$$

and thus depend on the complete $N$ dof model and not only on the considered $i$ th mode, as $C_{\mathrm{p} i}$ does. In practice, $C_{0}>C_{\mathrm{p} i}>C_{\infty}$, depending on the considered mode, so that the stability limits in terms of $\beta$ are smaller but close to one. We can notice from figure 2 that the $\beta^{\text {inst }}$ coefficients for the series and parallel cases are different. Moreover, the closer $C_{\mathrm{p} i}$ is to $C_{\infty}$ (parallel) or $C_{0}$ (series), the higher the stability threshold on the value of $\beta$ is (i.e. the higher the value of $\beta^{\text {inst }}$ is) and the the higher the attenuation performance is. Hence, the maximum achievable value of $\beta$ for the two NC configurations depends on the mode considered. Two extreme cases are worth taking into account:

- according to equation (16), if the considered mode is in the low frequencies of the EMS spectrum, $C_{\mathrm{p} i}$ will be closer to $C_{0}$ than to $C_{\infty}$, so that the stability limit for the series configuration $\left(C_{2}>C_{0} \simeq C_{\mathrm{p} i}\right)$ will be reached for values of $\beta_{2}$ close to 1 ( $\left.\beta_{2}^{\text {inst }} \simeq 1\right)$, whereas the stability limit for the parallel configuration $\left(C_{1}<C_{\infty}<C_{\mathrm{p} i}\right)$ will be reached for values of $\beta_{1}$ far from $1\left(\beta_{1}^{\text {inst }}<1\right)$. According to figure 6 , in this case the series configuration will perform better than the parallel configuration;

- the opposite situation holds for high-frequency modes: the parallel configuration will theoretically perform better than the series one $\left(\beta_{1}^{\text {inst }} \simeq 1, \beta_{2}^{\text {inst }}<1\right)$.

To fix the ideas, we present an example considering an arbitrary EMS with 10 degrees of freedom. This example will allow us to explain clearly when it is convenient to use either the parallel or the series configuration, and when the SP becomes highly efficient if compared to these traditional NC layouts. In the example, the modes of the EMS are assumed to have the same MEMCF value, which is $k_{i}=0.1$. The values 
Table 2. Parameters of the EMS enhanced by NCs in parallel, series or series+parallel configurations, as a function of the stability margin parameter $\beta$.

\begin{tabular}{|c|c|c|c|c|c|}
\hline & & R-shunt & Parallel config. & Series config. & SP config. \\
\hline SC freq. & $\omega_{i}^{\mathrm{sc}}$ & $\omega_{i}$ & $\omega_{i}$ & $\omega_{i} \sqrt{1-\frac{\beta_{2} k_{i}^{2}}{1-\beta_{2}}}$ & $\omega_{i} \sqrt{1-\frac{\beta_{\mathrm{sp} 2} k_{i}^{2}}{1-\beta_{\mathrm{sp} 2}}}$ \\
\hline OC freq. & $\omega_{i}^{\mathrm{oc}}$ & $\hat{\omega}_{i}=\omega_{i} \sqrt{1+k_{i}^{2}}$ & $\omega_{i} \sqrt{1+\frac{k_{i}^{2}}{1-\beta_{1}}}$ & $\hat{\omega}_{i}=\omega_{i} \sqrt{1+k_{i}^{2}}$ & $\omega_{i} \sqrt{1+\frac{k_{i}^{2}}{1-\beta_{\mathrm{sp} 1}}}$ \\
\hline EMEMCF & $\tilde{k}_{i}$ & $k_{i}$ & $\frac{k_{i}}{\sqrt{1-\beta_{1}}}$ & $\frac{k_{i}}{\sqrt{1-\beta_{2}}}$ & 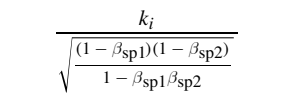 \\
\hline Elec. time const. & $\tau_{e}$ & $R_{\mathrm{sh}} C_{\mathrm{p} i}$ & $R_{\mathrm{sh}} C_{\mathrm{p} i}\left(1-\beta_{1}\right)$ & $\frac{R_{\mathrm{sh}} C_{\mathrm{p} i}}{1-\beta_{2}}$ & $\frac{R_{\mathrm{sh}} C_{\mathrm{p} i}\left(1-\beta_{\mathrm{sp} 1}\right)\left(1-\beta_{\mathrm{sp} 1} \beta_{\mathrm{sp} 2}\right)}{1-\beta_{\mathrm{sp} 2}}$ \\
\hline
\end{tabular}

Table 3. Value of $C_{\mathrm{p} i}$ and stability limits in term of the $\beta$ indexes and corresponding coupling factor enhancement $\tilde{k}_{i} / k_{i}$ for an arbitrary EMS with ten natural modes, with $k_{i}=0.1$ for all the ten modes.

\begin{tabular}{|c|c|c|c|c|c|c|c|}
\hline & \multirow[b]{2}{*}{$C_{\mathrm{p} i} / C_{0}$} & \multirow[b]{2}{*}{$C_{\mathrm{p} i} / C_{\infty}$} & \multicolumn{2}{|c|}{ Parallel config. } & \multicolumn{2}{|c|}{ Series config. } & \multirow{2}{*}{$\frac{\text { SP config }}{\tilde{k}_{i} / k_{i}}$} \\
\hline & & & $\beta_{1}^{\text {inst }}$ & $\tilde{k}_{i} / k_{i}$ & $\beta_{2}^{\text {inst }}$ & $\tilde{k}_{i} / k_{i}$ & \\
\hline mode 1 & $1 / 1.01$ & 1.09 & 0.92 & 3.56 & 0.99 & 10 & 10.56 \\
\hline mode 5 & $1 / 1.05$ & 1.05 & 0.95 & 4.47 & 0.95 & 4.47 & 6.24 \\
\hline mode 9 & $1 / 1.09$ & 1.01 & 0.99 & 10 & 0.92 & 3.56 & 10.56 \\
\hline
\end{tabular}

of $C_{\mathrm{p} i}$ (equation (14)), as compared to $C_{0}$ (equation (15)) and $C_{\infty}$, are gathered in table 3 for the first, the fifth and the ninth mode. As for the first mode, the series configuration works better than the parallel one at the stability limit because the first mode is in the low frequency part of the spectrum. Indeed, the series configuration is able to increase the coupling factor by a factor of 10 , whereas the parallel one allows us to achieve a value of EMEMCF just 3.56 times the original MEMCF. As for the ninth mode, located in the upper part of the frequency spectrum, the opposite result is observed. As for the fifth mode, the parallel and series configurations show the same performance: a coupling factor enhancement of a factor 4.47 is achieved by both the NC configurations. The performance of the SP configuration is also shown in table 3. Since its stability depends on two conditions, related to the two negative capacitances in series and in parallel, the best gain in using an SP configuration as compared to a single NC in parallel or series configuration is for the medium frequency modes: for mode 5, the SP configuration gives a coupling factor enhancement of 6.24 times instead of 4.47 (obtained with the traditional $\mathrm{NC}$ layouts).

\section{Optimisation and performance with ideal circuits}

Sections 2 and 3 described the mathematical model to treat EMSs shunted with impedances made up by NCs and $R_{\mathrm{sh}}$ as well as the effect of NCs on the MEMCF. This section is aimed at the derivation of the analytical procedures for defining the optimal values of the shunt impedance components (i.e. $\mathrm{NC}$ and $R_{\mathrm{sh}}$ ) in order to achieve the best vibration reduction, as well as for the quantification of the performance of the shunt in terms of vibration reduction.

\subsection{Tuning of the negative capacitance}

Section 3.3 showed that the closer the negative capacitances are to the stability limits, the higher the EMEMCF is. Therefore, the attenuation performance can be improved using negative capacitances as close as possible to the stability limits.

\subsection{Tuning of the resistance $R_{\mathrm{sh}}$ for single mode control}

The other component of the shunt circuit to be tuned is the resistance $R_{\mathrm{sh}}$. This section explains how to optimise it when single mode vibration reduction is required. The criterion proposed herein is based on considerations of the shape of the controlled FRF of the EMS.

If the mechanical system to damp shows low modal density, we can approximate the response of the EMS as the one given by the $i$ th mode. Therefore, it is possible to define the optimal value of $R_{\mathrm{sh}}$ by considering the FRF $H_{i}$ of equation (26), which is valid for the R-shunt without the NC [2] as well as for the parallel, series and SP configuration, in the case of a 1 dof approximation of the model (see section 2.5). This means that the procedure used to find the optimal value of $R_{\mathrm{sh}}$ for a traditional $\mathrm{R}$-shunt can be applied to the present cases with the negative capacitance. It will also provide results of the same form for the three layouts.

As suggested in $[1,2,18,19]$, the optimisation problem is solved by neglecting the structural damping $\xi_{i}$. Under such a hypothesis, there exists a point (here denoted by $F$ ) common to the amplitudes of all the FRFs of the EMS when $R_{\mathrm{sh}}$ is varied. Since $F$ is common to all curves describing the 
amplitude of the FRF, the optimum one (the one which has the lowest peak amplitude) is that with its maximum at point $F$. The frequency value $\omega_{F}$ associated with point $F$ can be obtained by remarking that, among all possible FRFs, this point is common to two particular cases: the one in the shortcircuit $\left(R_{\mathrm{sh}}=0\right.$ and $\left.\tau_{e}=0\right)$ and the one in the open-circuit $\left(R_{\mathrm{sh}}=+\infty\right.$ and $\left.\tau_{e}=+\infty\right)$, for which the FRF expression assumes a simple mathematical form. The frequency $\omega_{F}$ is found to be:

$$
\omega_{F}=\sqrt{\frac{\left(\omega_{i}^{\mathrm{oc}}\right)^{2}+\left(\omega_{i}^{\mathrm{sc}}\right)^{2}}{2}}
$$

where $\omega_{i}^{\text {oc }}$ and $\omega_{i}^{\text {sc }}$ are calculated depending on the configuration used (i.e. series, parallel, SP; see table 1). The associated optimal value for $\tau_{e}$ is:

$$
\tau_{e}^{\mathrm{opt}}=\frac{1}{\omega_{F}}
$$

The above reasoning is illustrated in figure 4 , where the location of point $F$ is shown with a bullet on the R, S, P and SP optimised curves (with $\tau_{e}=\tau_{e}^{\text {opt }}$ ), depending on the NC configuration considered. The above theoretical results are also validated since these optimised responses have their maximum at the crossing of the corresponding OC (i.e. $R_{\mathrm{sh}}=+\infty$ ) and SC (i.e. $R_{\mathrm{sh}}=0$ ) curves.

The above parameters $\tau_{e}^{\text {opt }}$ and $\omega_{F}$ can be rewritten in terms of $\tilde{k}_{i}$ by noticing that $\left(\omega_{i}^{\mathrm{oc}}\right)^{2}=\omega_{i}^{2} \tilde{k}_{i}^{2}+\left(\omega_{i}^{\mathrm{sc}}\right)^{2}$ (equation (23)) and by writing $\left(\omega_{i}^{\text {sc }}\right)^{2}=\omega_{i}^{2}\left(1-K^{2}\right)$, with $K$ defined in table 4 . One obtains:

$$
\omega_{F}=\frac{1}{\tau_{e}^{\mathrm{opt}}}=\omega_{i} \sqrt{\frac{\tilde{k}_{i}^{2}+2-2 K^{2}}{2}} .
$$

Finally, the optimal value of $R_{\mathrm{sh}}$ can be obtained by using the above equation together with the last row of tables 1 or 2 .

\subsection{Performance evaluation for single mode control}

The performance of the shunts is evaluated by defining the vibration attenuation parameter $A_{\mathrm{dB}}$, proposed in $[2,20]$ for passive shunts, as:

$$
A_{\mathrm{dB}}=20 \log _{10} \frac{H_{\mathrm{sc}}}{H_{\mathrm{sh}}}
$$

where $H_{\mathrm{sc}}$ is the FRF peak amplitude in short-circuit configuration without any shunt (i.e. uncontrolled EMS) and $H_{\mathrm{sh}}=\left|H_{i}\right|_{\Omega=\omega_{F}, \tau_{e}=\tau_{e}^{\text {opt }}}$ is the FRF peak amplitude with the optimised shunt (with $\tau_{e}=\tau_{e}^{\mathrm{opt}}$ ) connected. $A_{\mathrm{dB}}$ is shown in figure 7. Since $H_{\mathrm{sc}}=1 /\left(2 \xi_{i} \omega_{i}^{2} \sqrt{1-\xi_{i}^{2}}\right)$, after some symbolic manipulations, one obtains a single expression of $A_{\mathrm{dB}}$ valid for all four shunt layouts studied in this paper:

$$
A_{\mathrm{dB}}=20 \log _{10} \frac{\tilde{k}_{i}^{2}+2 \sqrt{2} \xi_{i} \sqrt{2+\tilde{k}_{i}^{2}-2 K^{2}}}{4 \xi_{i} \sqrt{1-\xi_{i}^{2}}}
$$

where the enhanced coupling factor $\tilde{k}_{i}$ takes the values of the third rows of tables 1 and 2 depending on the configuration used. The values of $K$ are gathered in table 4 .

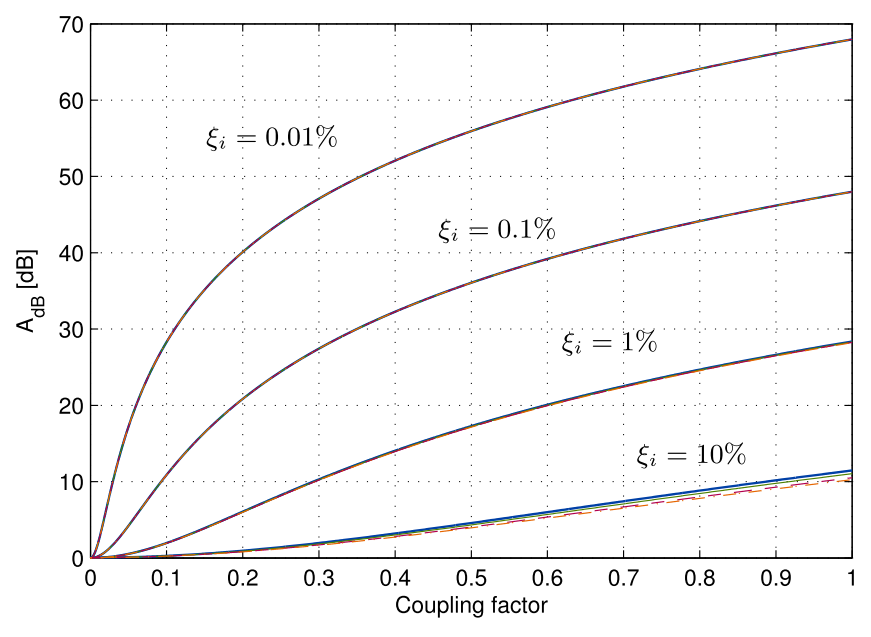

Figure 7. Attenuation $A_{\mathrm{dB}}$ achievable with the four shunt layouts as a function of the coupling factor $k_{i}$ (for the R-shunt) and of the enhanced coupling factor $\tilde{k}_{i}$ for the shunts with NC (parallel, series and SP), for several values of structural damping $\xi_{i}$. For each value of $\xi_{i}$, four curves are merged, one for each configuration: parallel, series, SP and simple resistive shunt.

The first major result from equation (43) is that, for a negative capacitance shunt in parallel configuration, $A_{\mathrm{dB}}$ has the same form as the one for a standard R-shunt, provided $k_{i}$ is replaced by the enhanced coupling factor $\tilde{k}_{i}$. As for the series and SP configuration, a slight difference is brought about by the term $K^{2}$. In these two latter situations, by noticing (tables 2 and 4 ) that $K$ and $\tilde{k}_{i}$ are functions of $k_{i}$ and $\beta$ only, $A_{\mathrm{dB}}$ can be expressed as a function of $\tilde{k}_{i}$ and $\beta_{2}$ (or $\beta_{\mathrm{sp} 2}$ ) only. To evaluate the effect of $K^{2}$, figure 7 shows $A_{\mathrm{dB}}$ as a function of $\tilde{k}_{i}$ for several values of $\xi_{i}$. It shows that, for each value of $\xi_{i}$, all the curves are almost merged, so that in practice the effect of the term $K^{2}$ can be neglected: for a negative capacitance shunt, $A_{\mathrm{dB}}$ has the same form as the one for a standard $\mathrm{R}$-shunt, provided $k_{i}$ is replaced by the enhanced coupling factor $\tilde{k}_{i}$. This result is very interesting in practice since it enables us to estimate the performance of the negative shunts relying only on two simple graphs. Indeed, it is sufficient:

- to know the values of the MEMCF $k_{i}$ and the damping factor $\xi_{i}$ for the considered mode;

- to know the margin from the stability limit of the considered negative capacitance shunt (the value of $\beta$, see section 3.3). Then, figure 6 enables us to estimate the increase in the coupling factor, from $k_{i}$ to $\tilde{k}_{i}$;

- then, figure 7 leads us to estimate the resonance attenuation brought about by the considered shunt as a function of $\tilde{k}_{i}$ and $\xi_{i}$.

In the same manner as for a standard R-shunt (see [2]), the higher the coupling factor $k_{i}$ is and the lower the structural damping $\xi_{i}$ is, the better the attenuation performances are.

Another way of evaluating the shunt performances is to consider the magnification factor at resonance $D$ - that is the ratio between the resonance peak amplitude $H_{\mathrm{sh}}$ and the static (for $\Omega=0$ ) amplitude of the FRF $\left(H_{i}(\Omega=0)=1 /\left(\omega_{i}^{\text {sc }}\right)^{2}\right)$. Figure 8 shows $D$ as a function of $\tilde{k}_{i}$. An interesting point is 
Table 4. Definition of $K$.

\begin{tabular}{lccc}
\hline R-shunt & Parallel & Series & SP \\
\hline$K=0$ & $K=0$ & $K=\frac{\chi_{i}}{\omega_{i} \sqrt{C_{2}-C_{\mathrm{p} i}}}=k_{i} \sqrt{\frac{\beta_{2}}{1-\beta_{2}}}$ & $K=\frac{\chi_{i}}{\omega_{i} \sqrt{C_{1}+C_{2}-C_{\mathrm{p} i}}}=k_{i} \sqrt{\frac{\beta_{\mathrm{sp} 2}-\beta_{\mathrm{sp} 2}}{2}}$ \\
\hline
\end{tabular}

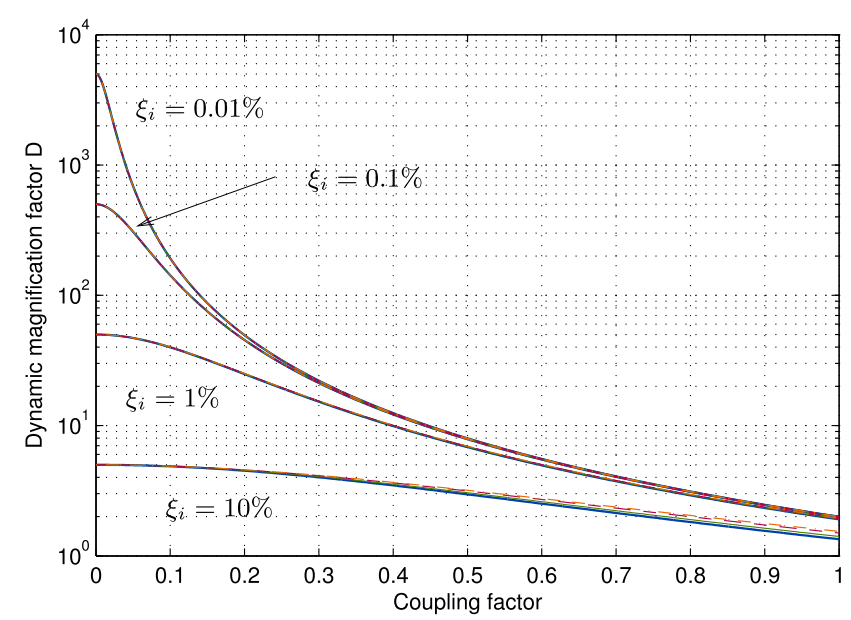

Figure 8. The magnification factor at resonance $D$ due to the four shunt layouts as a function of the coupling factor $k_{i}$ (for the R-shunt) and of the enhanced coupling factor $\tilde{k}_{i}$ for the shunts with NC (parallel, series and SP), for several values of structural damping $\xi_{i}$. For each value of $\xi_{i}$, four curves are merged, one for each configuration: parallel, series, SP and simple resistive shunt.

that for low structural damping $\xi_{i}$ and a high coupling factor, several $D$ curves are merged, so that $D$ only slightly depends on $\xi_{i}$ (for instance, the curves for $\xi_{i}=0.1 \%$ and $\xi_{i}=0.01 \%$ are merged for $\tilde{k}_{i}>0.2$ ). As a consequence, with large coupling factors achievable using NCs, the amplitude at resonance depends mainly on the coupling factor and no longer on the damping factor.

\subsection{Tuning of the resistance $R_{\text {sh }}$ and performance evaluation for multi-mode control}

When multi-mode control is required, numerical minimisations can be carried out in order to find the value of $R_{\mathrm{sh}}$ which fulfills the desired goal (e.g. $H_{\infty}$ control, $H_{2}$ control). This section explains how to find the value of $R_{\mathrm{sh}}$ without carrying out any numerical minimisation and thus avoiding any possible problems relating to minimisation procedures (e.g. local minima). This goal will be achieved by means of an easy-toapply graphical approach relying on an analytical formulation, as explained underneath.

For a given value of $R_{\mathrm{sh}}$ (and thus of $\tau_{e}$ ), we call $\omega_{i}^{\mathrm{r}}$ the resonance frequency of mode $i$, i.e. the frequency for which the FRF modulus $\left|H_{i}\right|$ reaches its maximum. We have $\omega_{i}^{\text {sc }}<\omega_{i}^{\mathrm{r}}<\omega_{i}^{\text {oc }}$ when $\tau_{e}$ is varied. This resonance frequency $\omega_{i}^{\mathrm{r}}$ can be calculated by solving the following equation as a function of $\Omega$ :

$$
\frac{\partial\left|H_{i}(\Omega)\right|^{2}}{\partial \Omega}=0
$$

Once $\omega_{i}^{\mathrm{r}}\left(\tau_{e}, \omega_{i}\right)$ is known as a function of $\tau_{e}$, the amplitude at the resonance can be derived with equation (26):

$$
H_{i}^{\max }=\left|H_{i}\left(\Omega=\omega_{i}^{\mathrm{r}}\right)\right| .
$$

It must be noted that the performance indicator $A_{\mathrm{dB}}$ (equation (42)) is related to the value of $H_{i}^{\max }$ for $\tau_{e}=\tau_{e}^{\mathrm{opt}}$

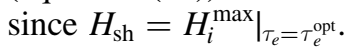

To calculate $\omega_{i}^{\mathrm{r}}$, equation (44) gives (if $\xi_{i}$ is considered null, as done when calculating $\omega_{F}$ ) the following seventh order equation in $\Omega$ :

$$
\Omega\left(a \Omega^{6}+b \Omega^{4}+c \Omega^{2}+d\right)=0
$$

where

$a=2 \tau_{e}^{4}, \quad b=4 \tau_{e}^{2}-2\left(\omega_{i}^{\mathrm{oc}}\right)^{2} \tau_{e}^{4}$,

$c=2-4\left(\omega_{i}^{\mathrm{oc}}\right)^{2} \tau_{e}^{2}, \quad d=\left(\omega_{i}^{\mathrm{oc}}\right)^{4} \tau_{e}^{2}-\left(\omega_{i}^{\mathrm{sc}}\right)^{4} \tau_{e}^{2}-2\left(\omega_{i}^{\mathrm{sc}}\right)^{2}$

The terms $\omega_{i}^{\text {sc }}$ and $\omega_{i}^{\text {oc }}$ must be calculated according to the configuration used (see table 1).

Equation (46) leads to the trivial solution $\Omega=0$ and to three other possible solutions for $\Omega^{2}$. The third order equation in $\Omega^{2}$ can be solved analytically by using the formula found by Tartaglia [21]. Let us define:

$$
\begin{aligned}
p= & c / a-b^{2} /\left(3 a^{2}\right), \quad m=d / a-b c /\left(3 a^{2}\right) \\
& +2 b^{3} /\left(27 a^{3}\right), \quad \Delta=m^{2} / 4+p^{3} / 27
\end{aligned}
$$

If $\Delta<0$, the solutions of the cubic equation in $\Omega^{2}$ (see equation 46) are:

$$
\begin{gathered}
\Omega_{1}^{2}=2 \sqrt{-p / 3} \cos [\vartheta / 3]-b /(3 a) \\
\Omega_{2}^{2}=2 \sqrt{-p / 3} \cos [(\vartheta+2 \pi) / 3]-b /(3 a) \\
\Omega_{3}^{2}=2 \sqrt{-p / 3} \cos [(\vartheta+4 \pi) / 3]-b /(3 a)
\end{gathered}
$$

where $\vartheta=\arctan [\sqrt{-\Delta} /(-m / 2)]$ and $\arctan$ must be intended as the four-quadrant arctangent. If $\Delta \geqslant 0$, the solutions are not provided here because when $\Delta$ is higher than or equal to zero, the attenuation provided by the shunt is usually so high that the resonance is canceled by the control action. As a result, the maximum of $\left|H_{i}\right|$ between $\omega_{i}^{\text {sc }}$ and $\omega_{i}^{\text {oc }}$ is lower than its static component (i.e. $\left.\left|H_{i}(\Omega=0)\right|=1 /\left(\omega_{i}^{\mathrm{sc}}\right)^{2}\right)$, and the only physical solution provided by equation (46) is $\omega_{i}^{\mathrm{r}}=0^{5}$. Therefore, if the

5 This result was checked by means of simulations with $2 \pi 10 \mathrm{rad} / \mathrm{s}<\omega_{i}<2 \pi 3000 \mathrm{rad} / \mathrm{s}, \quad 0.001<k_{i}<0.3, \quad 0<\beta<1$ and $\tau_{e}^{\text {opt }} / 100<\tau_{e}<100 \tau_{e}^{\text {opt }}$. We chose the simulation values in order to cover most of the engineering applications. 


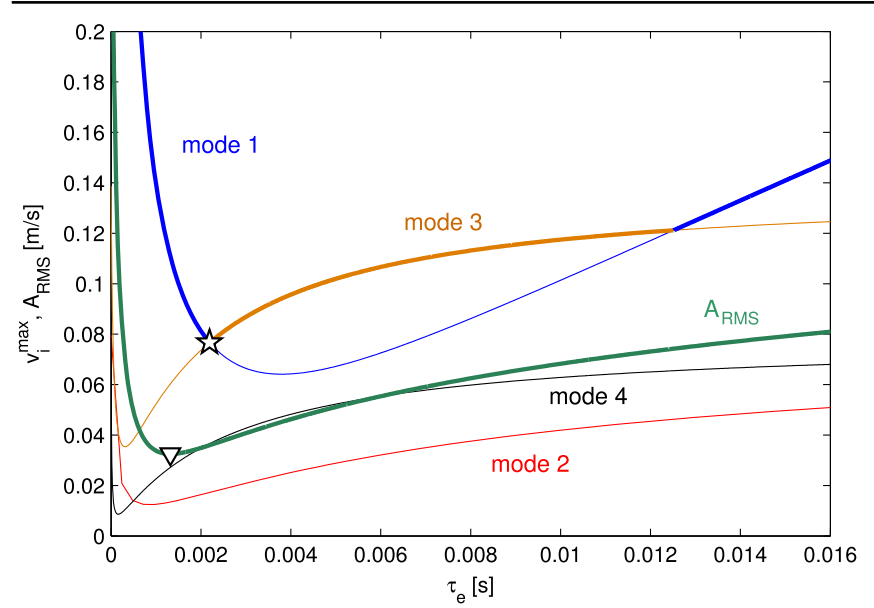

Figure 9. The trend of $v_{i}^{\max }$ and $A_{\mathrm{RMS}}$ for the four modes of the EMS described in table 5 for an NC in series configuration. $C_{\mathrm{p} i}=30.5 \mathrm{nF}$ and $C_{2}=36.6 \mathrm{nF}$. The values of the mode shape components used here are: $\Phi\left(x_{f}\right)=[1.7167,0.2450,0.8020,1.1987]^{\prime}$ and

$\Phi\left(x_{m}\right)=[2.3452,2.2935,-2.8761,-2.2822]^{\prime}$.

Table 5. Modal data of the system used for the numerical simulations and for the experimental tests.

\begin{tabular}{lccc}
\hline Mode number & $\omega_{i} /(2 \pi)[\mathrm{Hz}]$ & $\xi_{i}$ & $k_{i}$ \\
\hline 1 & 39.72 & 0.0045 & 0.2716 \\
2 & 187.34 & 0.0030 & 0.1037 \\
3 & 509.00 & 0.0026 & 0.0710 \\
4 & 986.42 & 0.0028 & 0.1222 \\
\hline
\end{tabular}

chosen value of $\tau_{e}$ gives a value of $\Delta$ higher than or equal to zero, the maximum of the amplitude of $H_{i}$ can be approximated by its static component. If the chosen value of $\tau_{e}$ gives a value of $\Delta$ lower than zero, the three solutions (49a)-(c) must be taken into consideration. Only one of them has a physical meaning and it is found to be $(49 a){ }^{5}$ If a different situation with respect to the cases considered in footnote 5 is taken into account, and equation (49a) does not provide a physical solution, solutions (49b) and (49c) must be considered, because one of them will provide the correct one.

Therefore, the curves relating the value of $R_{\mathrm{sh}}$ to $H_{i}^{\max }$ can be drawn for all the modes to be controlled. This gives us the possibility of finding out the optimal value of $R_{\mathrm{sh}}$ for a given control problem with a graphical approach, as explained in the following example. Let us consider the system described in table 5 (similar to that used for experimental tests in the following), an NC in series and a $H_{\infty}$ control problem on the velocity of a given point of the EMS. If $x_{m}$ is a point where the structural response is measured and $x_{f}$ is a point where an excitation force is provided, the FRF between this force and the displacement of the structure in $x_{m}$ is obtained by multiplying equation (26) by $\Phi_{i}\left(x_{m}\right) \Phi_{i}\left(x_{f}\right)$. Hence, for each of the modes considered it is possible to draw the curve relating $R_{\mathrm{sh}}$ to the maximum of the velocity FRF:

$$
v_{i}^{\max }=\left|\Phi_{i}\left(x_{f}\right) \Phi_{i}\left(x_{m}\right) \omega_{i}^{\mathrm{r}} H_{i}^{\max }\right|
$$

The resulting curves are plotted in figure 9 . In this figure, the graph has $\tau_{e}$ on the $x$-axis in place of $R_{\mathrm{sh}}$ because $C_{\mathrm{p} i}$ was fixed to the same value for all four modes (indeed the values of $C_{\mathrm{p} 1}$ to $C_{\mathrm{p} 4}$ were so close that an average value has been used) and thus a given value of $R_{\mathrm{sh}}$ corresponds to the same value of $\tau_{e}$ for all the modes. The portions of the solid curves with an increased width (blue and orange in figure 9) are those providing evidence of the highest peak of the velocity FRF for the EMS as a function of $\tau_{e}$. The minimum of this line corresponds to the value of $R_{\mathrm{sh}}$ to be chosen if an $H_{\infty}$ control must be performed. This figure clearly shows the optimal value of $\tau_{e}$ without the need to carry out numerical minimisations, and evidence of this optimal value is provided by a star in the figure. The same procedure can be applied to acceleration as well, just by multiplying equation (50) by $\omega_{i}^{\mathrm{r}}$.

This graphical approach can also lead us to find the optimal value of $R_{\mathrm{sh}}$ for other control problems. As an example, for an $\mathrm{H}_{2}$ control in velocity (i.e. minimisation of the velocity root mean square value, RMS), one can consider minimising the following approximated quantity:

$$
A_{\mathrm{RMS}}=\sqrt{\sum_{i=1}^{N} \frac{\left(v_{i}^{\max }\right)^{2}}{2}}
$$

The minimum of this curve (solid green curve in figure 9), highlighted by a triangle in figure 9 , shows which value of $\tau_{e}$ makes the RMS minimum. Note that the $H_{\infty}$ control (star) and the $\mathrm{H}_{2}$ control (triangle) require different solutions, which can easily be found graphically, and the optimal values of $\tau_{e}$ for the $H_{2}$ and $H_{\infty}$ problems do not correspond to the optimal values of the four modes (minima of the solid thin lines of figure 9).

This graphical approach works with FRFs, but it even allows us to solve problems where the input disturbance is not white random noise. Indeed, if the power-spectrum $G_{d d}$ of the disturbance can be estimated, the control problem can be expressed not in terms of FRF but in terms of power-spectrum of the EMS response $G_{r r}$, which can be calculated in case of low modal density as:

$$
G_{r r}\left(\omega_{i}^{\mathrm{r}}\right)=G_{d d}\left(\omega_{i}^{\mathrm{r}}\right)\left|\Phi_{i}\left(x_{m}\right) \Phi_{i}\left(x_{f}\right) H_{i}\left(\omega_{i}^{\mathrm{r}}\right)\right|^{2}
$$

\section{Real circuits}

\subsection{Negative capacitance layouts}

Section 1 mentioned that an NC can be built using OP-AMPs and that we distinguish two different types: ideal circuits (ICs), which can be modelled as pure NCs, and more complicated circuits (real circuits, RCs), which cannot be modelled as pure negative capacitances. RCs are often used in practice because they offer significant advantages when compared to ICs. Indeed, they are able to overcome some of the main issues relating to ICs. All the circuits are discussed in this section.

A negative impedance can be built in practice by using an OP-AMP along with three different impedances 
(a)

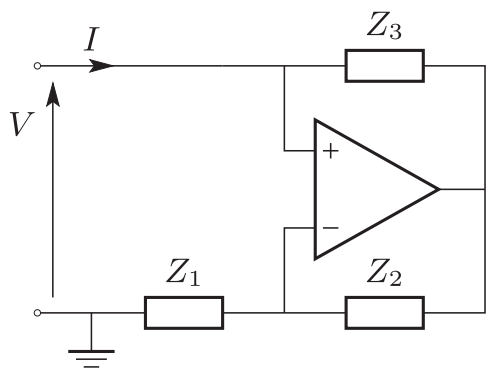

Figure 10. A negative impedance built by means of an OP-AMP (a) and its electrical model (b).

(i.e. $Z_{1}, Z_{2}, Z_{3}$ ), as shown in figure 10 . The impedance of this circuit (the ratio between voltage and current intensity $\underline{V} / \underline{I}$ ) is:

$$
Z_{\mathrm{nc}}=\frac{-Z_{1} Z_{3}}{Z_{2}}
$$

Equation (53) is valid as long as the OP-AMP is considered ideal, which in turn means that the voltage difference between the inputs is null and no current flows in the inputs [15]. $Z_{\text {nc }}$ becomes an NC if $Z_{1}, Z_{2}$ and $Z_{3}$ are replaced by resistances and a capacitance respectively. In order to obtain a pure negative capacitance-as in figure 3(c)-two different configurations are possible, labelled type A and type B circuits, as shown in figures 11(a), (b). In this case, we have an equivalent negative capacitance $-C_{n}$ with:

$$
C_{\mathrm{n}}=\frac{R_{2} \hat{C}}{R_{1}}
$$

and the corresponding circuits are denoted as ideal circuits (ICs).

Furthermore, some authors suggest adding a resistance $\hat{R}$ in parallel to the capacitance $\hat{C}$ [22], leading to the circuits of figures 11(c) and (d) (types AR and BR). These circuits, denoted as real circuits (RCs) in the following, are not pure NCs: their impedance $Z_{\mathrm{nc}}$ is equivalent to a pure $\mathrm{NC},-C_{\mathrm{n}}$, (see equation (54)) in parallel with a negative resistance, $-\tilde{R}$, with:

$$
\tilde{R}=\frac{R_{1} \hat{R}}{R_{2}}
$$

The parallel between $\hat{C}$ and $\hat{R}$ constitutes an undesired but necessary high-pass filter useful for solving practical problems such as bias-current and offset-voltage induced errors [22].

The addition of $\hat{R}$ is often necessary when a series configuration is taken into consideration. Instead, in the parallel configuration, the parallel between the capacitance of the piezoelectric actuator and $R_{\mathrm{sh}}$ (see section 5.2) already constitutes a high-pass filter. Nonetheless, the addition of $\hat{R}$ can be useful even in this case (as found in the experiments related to this paper) because the values of the capacitance of the piezoelectric actuator and $R_{\mathrm{sh}}$ can be unsuitable for properly stopping the low-frequency components. In such cases, it is useful to add $\hat{R}$ in order to produce a further parallel between $\hat{C}$ and $\hat{R}$ and thus a more efficient high-pass filter. Moreover, the polarity of the OP-AMP is of practical importance (it has no influence in theory since equation (53) is the same whatever the $+/-$ input pin wiring be). As for a parallel configuration, the circuits of figure 11 can be used. For a series connection, the OP-AMP $+/-$ input pins must be inverted. Most of the circuits presented here and used to build an $\mathrm{NC}$ are employed in the literature, without providing any comparison between the different solutions. Table 6 shows how different authors have used different layouts.

The theoretical analyses carried out so far, in sections 2 , 3 and 4 , regard ICs without $\hat{R}$. The effect of adding $\hat{R}$ to the circuit on the behaviour of the shunt is now addressed.

\subsection{Parallel configuration for real circuits}

An RC in parallel configuration can be modelled as shown in figure 12(a). The total resistance in parallel to the piezoelectric actuator is the parallel between $R_{\mathrm{sh}}$ and $-\tilde{R}$ (due to the presence of $\hat{R}$, see equation (55)), which is named $R_{\mathrm{p}}$ :

$$
R_{\mathrm{p}}=\frac{-\tilde{R} R_{\mathrm{sh}}}{R_{\mathrm{sh}}-\tilde{R}}
$$

This total resistance $R_{\mathrm{p}}$ can be considered as the new shunt resistance, and thus the analytical treatment is the same as the ICs in a parallel configuration with just one difference: the resistance $R_{\mathrm{sh}}$ must be replaced by $R_{\mathrm{p}}$ in the equations of sections 2, 3 and 4. Hence, the RCs in the parallel configuration can be fully described by the model presented so far. Therefore, the stability of the EMS is guaranteed if the condition of equation (29) is fulfilled and $R_{\mathrm{p}} \geqslant 0$, and the tuning and performance of the shunt are those predicted in section 4 .

\subsection{Series configuration for real circuits}

An RC in a series configuration can be modelled as shown in figure 12(b). The impedance $Z_{\mathrm{rS}}$ of the circuit composed by $-C_{2},-\tilde{R}$ and $R_{\mathrm{sh}}$ in the Fourier domain is:

$$
Z_{\mathrm{rs}}=R_{\mathrm{sh}}-\frac{\tilde{R}}{1+j \Omega \tilde{R} C_{2}}
$$

where the values of $C_{2}$ and $\tilde{R}$ depend upon the values of $R_{1}$, $R_{2}, \hat{C}$ and $\hat{R}$ (see equations (54) and (55)). Generally speaking, this circuit cannot be treated with the formalism of sections 2, 3.3 and 4. Nevertheless, there are some cases where the effect of $\tilde{R}$ can be neglected, depending on the frequency band of interest, as shown in sections 5.3.1 and 5.3.2.

5.3.1. Similar behaviour of RCs and ICs. If $\hat{R}$-and thus $\tilde{R}-$ tend to $+\infty$, the impedance $Z_{\mathrm{rs}}$ tends to that of an IC that writes:

$$
Z_{\text {is }}=R_{\text {sh }}-\frac{1}{j \Omega C_{2}}
$$

In the high frequency range $\Omega \gg 1 /\left(\tilde{R} C_{2}\right)$, the effect of $\hat{R}$ can be neglected since $Z_{\mathrm{rs}} \simeq Z_{\text {is }}$ (see figure 13) and the behaviour of the real circuit is the one of a pure negative capacitance (in series with $R_{\mathrm{sh}}$ ). As a consequence, if all the controlled modes 
(a)

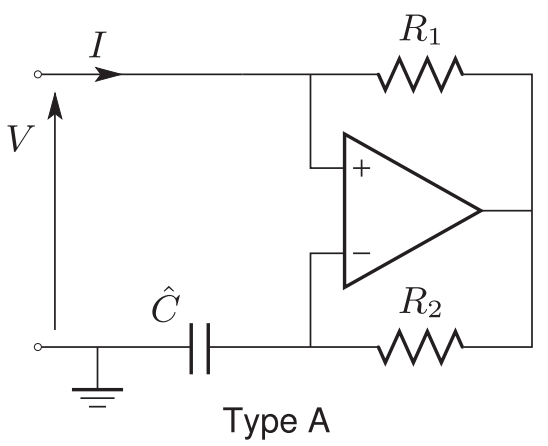

(c)

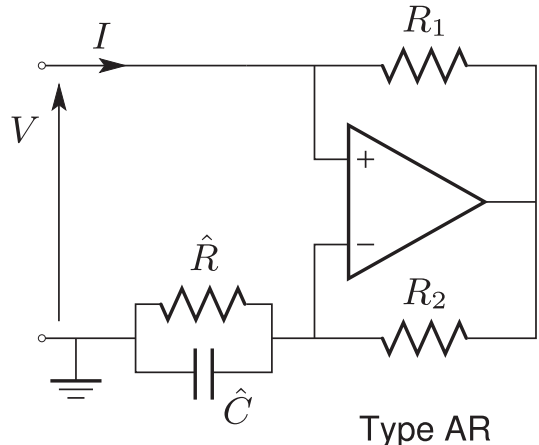

(b)

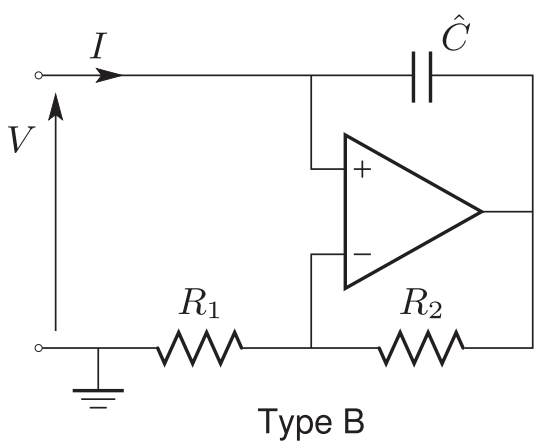

(d)

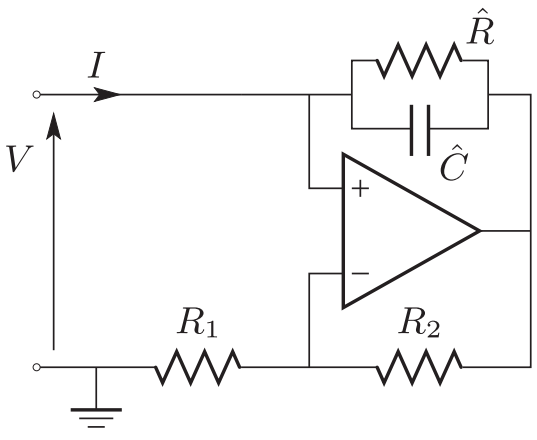

Type BR

Figure 11. Practical circuits to build a negative capacitance: type A (a), type B (b), type AR (c) and type BR (d) circuits in parallel configuration. For the series configuration, the pins of the OP-AMP must be exchanged.

Table 6. The NC layouts used in the literature, defined in figure 11. All series configurations have the OP-AMP +/- input pins exchanged with respect to figure 11 .

\begin{tabular}{ccc}
\hline Authors & Parallel configuration & Series configuration \\
\hline Behrens et al [4] & - & Type AR \\
Park and Baz [6] & Type B & - \\
de Marneffe and & Type B & Type B \\
Preumont [3] & & \\
Manzoni et al [7] & - & Type AR \\
Kodejška et al [8] & - & Type BR \\
Beck et al $[9,10]$ & - & Type BR \\
This paper & Type B (A, AR, BR & Type AR (A, B, BR \\
& also tested) & also tested) \\
\hline
\end{tabular}

of the EMS have their natural frequency above $1 /\left(\tilde{R} C_{2}\right)$, it is possible to use the results of sections $2,3.3$ and 4 without paying attention to $\hat{R}$.

It is possible to use the analytical formulation derived for ICs in a further case, even if the frequency range considered is below $1 /\left(\tilde{R} C_{2}\right)$. This is the case in which the value chosen for $R_{\mathrm{sh}}$ is high. In this case the values of $\tau_{e}^{\mathrm{opt}}, \omega_{i}^{\mathrm{r}}$ and $A_{\mathrm{dB}}$ obtained with $Z_{\mathrm{rs}}$ and $Z_{\text {is }}$ are usually very close. Such similar results for $Z_{\mathrm{rs}}$ and $Z_{\mathrm{is}}$ are shown in section 6.2 (see figure 17 in particular). The different behaviour between $Z_{\mathrm{rs}}$ and $Z_{\text {is }}$ becomes evident when $R_{\mathrm{sh}}$ is low, and in this case significant differences in the attenuation performance on the modes at low frequency become clear. Again, such a fact is shown in section 6.2. It is noticed that low values of $R_{\mathrm{sh}}$ mean that the control action is focused on the modes at high frequency.
5.3.2. Use of a compensation resistance. If the EMS shows natural frequencies to be controlled at low frequencies, below $1 /\left(\tilde{R} C_{2}\right)$, and if the value of $R_{\mathrm{sh}}$ we use is low, the analytical treatment used for ICs is not reliable for RCs. The IC and RC series circuits only differ for the presence of $-\tilde{R}$, which degrades the shunt performances. The effect of $-\tilde{R}$ can be cancelled by adding a further passive resistance $R_{\mathrm{S}}$ in parallel to $-\tilde{R}$ and $-C_{2}$, as shown in figure 12(c). The parallel between $R_{\mathrm{S}}$ and $-\tilde{R}$ (which is physically obtained by placing $R_{\mathrm{S}}$ between the upper connector of the impedances in figures 11(c), (d) and the ground) gives an equivalent resistance $R_{\text {eq }}$, defined as:

$$
R_{\mathrm{eq}}=\frac{-\tilde{R} R_{\mathrm{s}}}{R_{\mathrm{S}}-\tilde{R}}
$$

The aim is to achieve high negative values of $R_{\text {eq }}$ in order to cancel out the effect of $\tilde{R}$. Consequently, the required values of $R_{\mathrm{S}}$ should be close to $\tilde{R}\left(R_{\mathrm{S}} \simeq \tilde{R}\right)$, which produce high negative values of $R_{\mathrm{eq}}\left(\left|R_{\mathrm{eq}}\right| \rightarrow+\infty\right)$ and thus cancel out the effect of $\hat{R}$. In such a case, all the results of sections 2, 3.3 and 4 are valid and it is not necessary to consider $\hat{R}$. If the use of the compensation resistance $R_{\mathrm{S}}$ must be avoided for any practical reason (see section 6.2), a new analytical treatment can be developed for the shunt circuit in figure 12(b) (i.e. composed of $-C_{2},-\tilde{R}$ and $R_{\mathrm{sh}}$ ). This analytical treatment is given in appendix A.

\subsection{SP configuration for real circuits}

The SP circuit has been treated in section 3 and it is possible to deduce that this new configuration can be seen as an $\mathrm{NC}$ in 

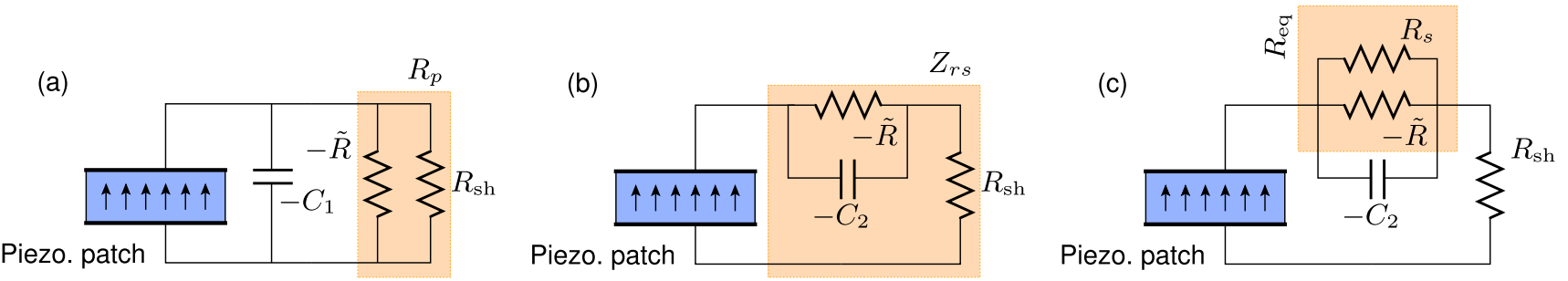

Figure 12. The equivalent model of the RC circuit for the parallel configuration (a); equivalent model of the RC circuit for the series configuration (b) and the addition of $R_{\mathrm{s}}$ (c).
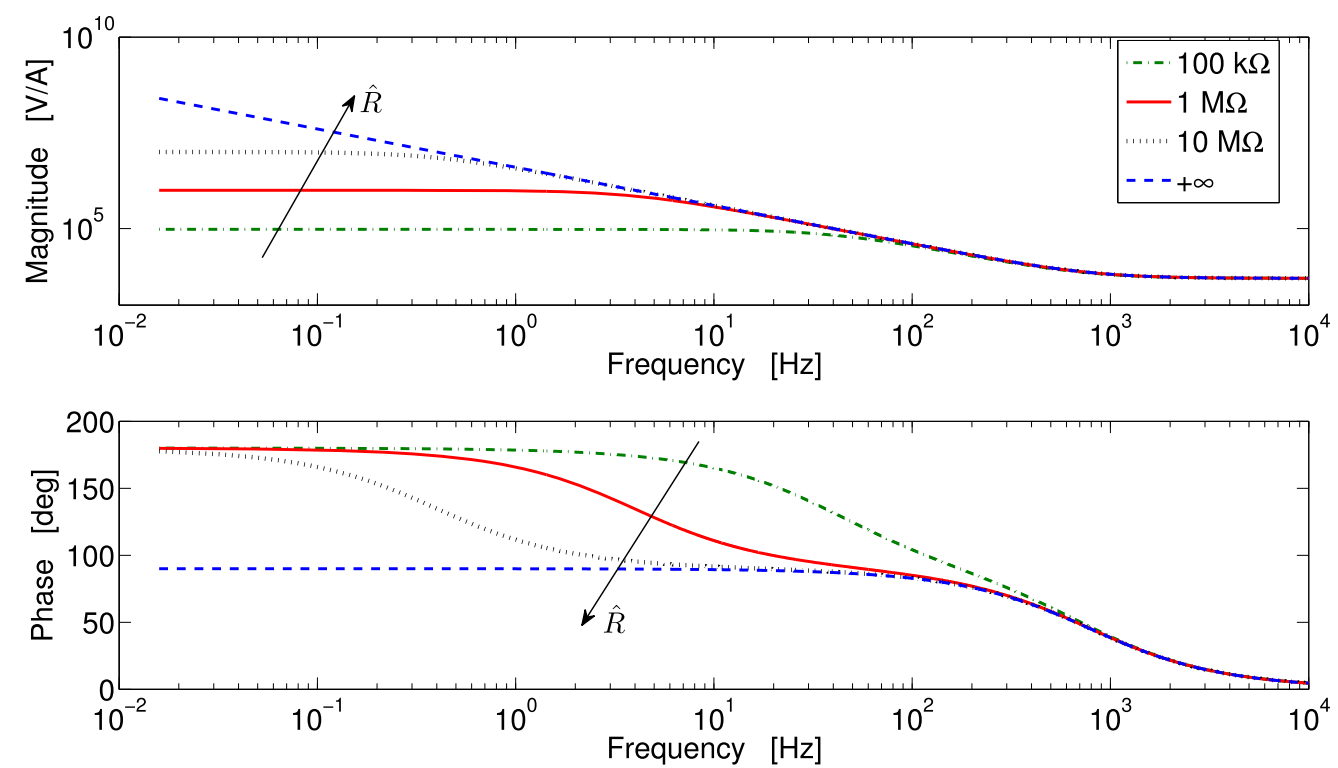

Figure 13. Magnitude and phase of the impedance $Z_{\mathrm{rs}}$ for different values of $\hat{R}$. In this figure: $R_{1}=R_{2}=5 \mathrm{k} \Omega, R_{\mathrm{sh}}=50 \mathrm{k} \Omega$ and $\hat{C}=40 \mathrm{nF}$. The case with $\hat{R}=+\infty$ corresponds to an IC (i.e. $Z_{\text {is }}$ ).

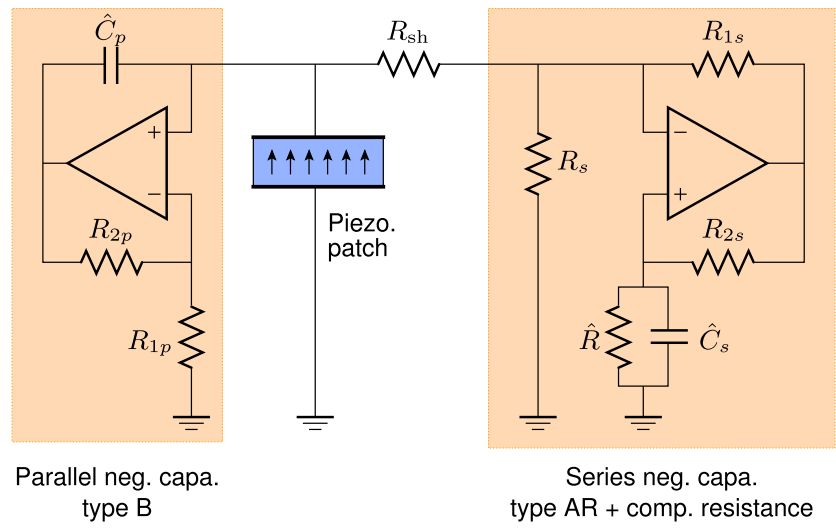

Figure 14. Electronic circuit used for the SP configuration.

series with a piezoelectric patch with a decreased capacitance (i.e. $C_{\text {eqp }}=C_{\mathrm{p} i}-C_{1}$, see figure 5 and equation (20)) as well as an enhanced MEMCF (i.e. $\tilde{k}_{i}=k_{i} / \sqrt{1-C_{\mathrm{l}} / C_{\mathrm{p} i}}$, see table 1). Hence, the description of the SP layout with RCs can be derived from sections 5.3 and 5.2. In this work, the RC SP circuit (see figure 14) is built using the IC configuration for $C_{1}$ (i.e. the parallel $\mathrm{NC}$ ) and an $\mathrm{RC}$ for $C_{2}$ (i.e. the series $\mathrm{NC}$ ).

\section{Experiments}

The analytical results shown previously were validated by a series of experiments. The test set-up was a stainless steel cantilever beam (with a length of $178.8 \mathrm{~mm}$, a width of $30.5 \mathrm{~mm}$ and a thickness of $1.1 \mathrm{~mm}$ ) with two PIC 151 piezoelectric patches (with a length of $70 \mathrm{~mm}$ each, $0.5 \mathrm{~mm}$ thick and with wrapped electrodes) bonded as depicted in figure 15. The modes considered for the tests were the first four bending modes, whose characteristics are given in table 5 (the shapes of these modes are the usual ones for cantilever beams). The values of $\omega_{i}$ and $\xi_{i}$ were estimated by means of experimental modal analysis and the values of $k_{i}$ were estimated by deriving the $\omega_{i}$ and $\hat{\omega}_{i}$ values from the measured FRFs of the EMS in a short- and open-circuit and using equation (13). Note that the values of table 5 are just indicative values. Indeed, the tests described from here on were carried out over several months. Therefore, small changes to the modal data were faced each time (e.g. due to thermal shift) and as a result, all the modal data were estimated before each test session. The values of the modal parameters relating to each kind of test discussed further on in the paper will always be given explicitly. The cantilever beam was excited by a 

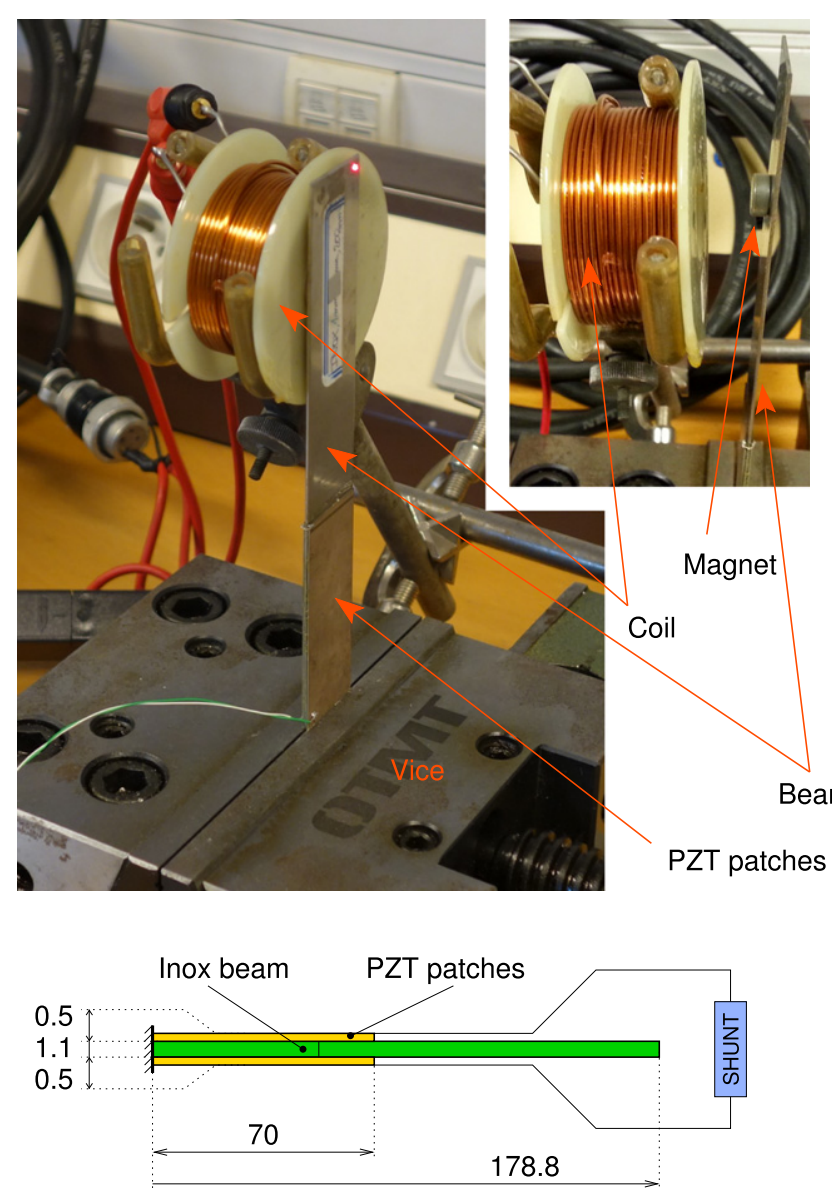

Figure 15. Experimental set-up. Dimensions are in millimetres.

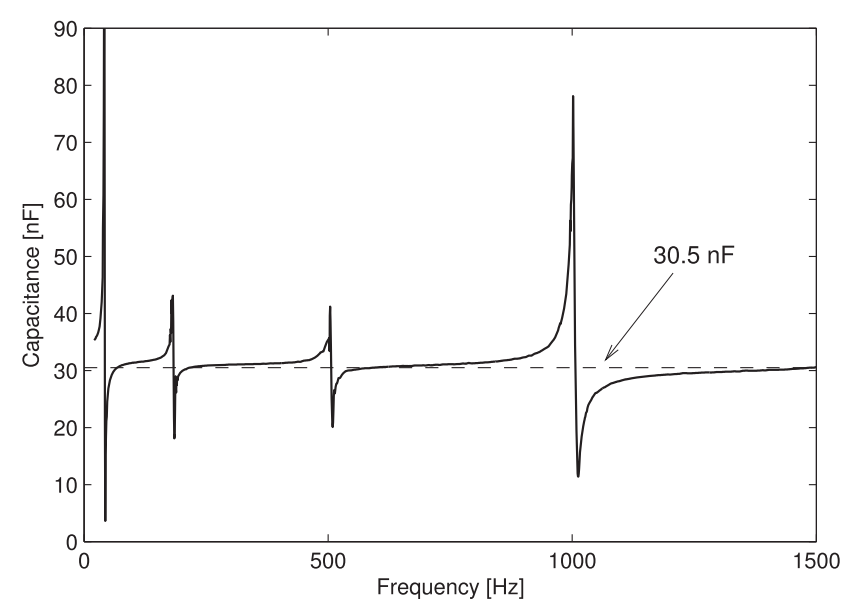

Figure 16. Experimental capacitance $C(\Omega)$ as a function of frequency of the piezoelectric patches in series.

contactless electro-magnetic actuator described in [23] composed by a fixed coil and a magnet bonded to the beam close to its tip (see figure 15). The vibrational response of the beam was measured by a laser Doppler velocimeter (Polytec PSV 400). The reference signal for the FRF computation was the intensity of the current that flowed in the coil, measured by a current clamp (Tektronix A622), assumed to be proportional to the electromagnetic force imposed on the magnet. A Brüel
\& Kjaer 2712 power amplifier was used to feed the coil. The NCs were built as proposed in figure 11 using Texas Instruments OPA445 operational amplifiers [24], with some of the resistances built using potentiometers for easy tuning. The chosen design of the circuits will be made clear in the following.

\subsection{Piezoelectric patch capacitance}

The two piezoelectric patches were electrically linked in series. The equivalent capacitance $C(\Omega)$ of both patches in series was measured as a function of frequency with a network analyser (NA). The voltage signal generated by the NA was sent to the terminals of the piezoelectric patches in series with a resistance $R=120 \mathrm{k} \Omega$, and the current intensity in the circuit was measured by monitoring the voltage at the terminals of $R$, thus obtaining the impedance $Z_{p}$ of the piezoelectric patches with the NA. The equivalent capacitance was then obtained by $C(\Omega)=1 /\left(j \Omega Z_{p}\right)$, as shown in figure 16 . The trend of $C(\Omega)$ is in full agreement with the theoretical one (shown in figure 2). Since it was not possible to obtain the limit response in the low-frequency range with this method, the plot has been deleted under $20 \mathrm{~Hz}$.

Estimating the values of $C_{0}, C_{\infty}$ and $C_{\mathrm{p} i}$ for $i=1, \ldots 4$ is not straightforward, since (i) $C_{0}$ and $C_{\infty}$ are the limits of $C(\Omega)$ in zero and high frequencies, which are not measured (ii) $C_{\mathrm{p} i}$ is the value of $C(\Omega)$ at an unknown frequency $\Omega$ between $\omega_{i}$ and $\omega_{i+1}$ (see section 2.2). A correct way of estimating $C_{0}, C_{\infty}$ and $C_{\mathrm{p} i}$ would be to update the theoretical model of equation (4) until its response matches the experimental one of figure 16. This has not been done in the present study.

Here, $C(\Omega)$ was measured at a number of frequency values between the first and the fifth mode of the structure with a digital multimeter. The results were close to one another: between 31.1 and $30.0 \mathrm{nF}$, which is in agreement with the values shown in figure 16. Since these values are very close each other, we decided to use a sort of average value for all the modes: $C_{\mathrm{p} i} \simeq C_{\mathrm{p}}=30.5 \mathrm{nF}$ for all of them ( $i=1,2,3,4)$, denoted by $C_{\mathrm{p}}$ in the following. $C_{0}$ was estimated by measuring the value of $C_{\mathrm{p} 1}$ at $100 \mathrm{~Hz}$ with a digital multimeter and then calculating $C_{0}=C_{\mathrm{p} 1}\left(1+k_{1}^{2}\right)$. The result for $C_{0}$ was about $33.26 \mathrm{nF}$. $C_{\infty}$ was estimated measuring the capacitance value at $100 \mathrm{kHz}$ and the result was about $25.9 \mathrm{nF}$.

\subsection{Tests with the compensation resistance}

This section shows that the use of a compensation resistance $R_{\mathrm{s}}$ allows $Z_{\mathrm{rs}}$ (i.e. $\mathrm{RC}$ in series, see figure $12(\mathrm{~b})$ ) to be made similar to $Z_{\text {is }}$ (i.e. IC in series) (see section 5.3). Different experiments were carried out with different configurations of the electrical parameters. Only one of them (employing type AR of figure 11) is taken into account here because all of the tests gave similar results. The values of the various parameters used for the experiment considered here are shown in table 7 (test 1 column). The value of $R_{\mathrm{S}}$ was changed step by step from $+\infty$ (i.e. the absence of $R_{\mathrm{s}}$ ) up to values which made $R_{\mathrm{eq}}$ closer and closer to $-\infty \quad\left(R_{s} \simeq \tilde{R}\right.$, see 
Table 7. Values of parameters for the different tests presented in the paper.

\begin{tabular}{|c|c|c|c|c|c|}
\hline Parameter & Test 1 & Test 2 & Test 3 & Test 4 & Test 5 \\
\hline$\omega_{1} /(2 \pi)[\mathrm{Hz}]$ & 39.72 & 39.71 & 39.69 & 39.84 & 39.72 \\
\hline$\hat{\omega}_{1} /(2 \pi)[\mathrm{Hz}]$ & 41.19 & 41.21 & 41.10 & 41.25 & 41.16 \\
\hline$\xi_{1}$ & 0.0038 & 0.0039 & 0.0040 & 0.0042 & 0.0045 \\
\hline$k_{1}$ & 0.2746 & 0.2774 & 0.2689 & 0.2778 & 0.2716 \\
\hline$\omega_{2} /(2 \pi)[\mathrm{Hz}]$ & 187.47 & 187.30 & 187.40 & 188.13 & 187.34 \\
\hline$\hat{\omega}_{2} /(2 \pi)[\mathrm{Hz}]$ & 188.42 & 188.48 & 188.35 & 189.15 & 188.34 \\
\hline$\xi_{2}$ & 0.0028 & 0.0033 & 0.0027 & 0.0035 & 0.0030 \\
\hline$k_{2}$ & 0.1008 & 0.1124 & 0.1008 & 0.1043 & 0.1037 \\
\hline$\omega_{3} /(2 \pi)[\mathrm{Hz}]$ & 509.53 & 508.92 & 509.10 & 510.15 & 509.00 \\
\hline$\hat{\omega}_{3} /(2 \pi)[\mathrm{Hz}]$ & 510.78 & 510.55 & 510.30 & 511.25 & 510.28 \\
\hline$\xi_{3}$ & 0.0032 & 0.0032 & 0.0026 & 0.0032 & 0.0027 \\
\hline$k_{3}$ & 0.0701 & 0.0801 & 0.0687 & 0.0657 & 0.0710 \\
\hline$\omega_{4} /(2 \pi)[\mathrm{Hz}]$ & 987.5 & 985.88 & 986.90 & 989.22 & 986.42 \\
\hline$\hat{\omega}_{4} /(2 \pi)[\mathrm{Hz}]$ & 994.84 & 994.34 & 994.10 & 996.00 & 993.75 \\
\hline$\xi_{4}$ & 0.0035 & 0.0036 & 0.0030 & 0.0035 & 0.0028 \\
\hline$k_{4}$ & 0.1222 & 0.1313 & 0.1210 & 0.1173 & 0.1222 \\
\hline$C_{1}, C_{2}[\mathrm{nF}]$ & 44.35 & see figure 18 & 19.82 & 49.10 & $\begin{array}{c}19.82 \text { (parallel) } \\
37.21 \text { (series) } \\
C_{1}=19.82 . C_{2}=1739(\mathrm{SP})\end{array}$ \\
\hline$\tilde{R}[\mathrm{M} \Omega]$ & 0.92 & $\begin{array}{c}1.00 \text { (for parallel RC) } \\
2.8 \text { (series) }\end{array}$ & - & 2.60 & $\begin{array}{c}C_{1}=19.82, C_{2}=17.39(\mathrm{SP}) \\
2.80(\text { series }) \\
2.80(\mathrm{SP}) \\
\text { - }(\text { parallel })\end{array}$ \\
\hline$R_{\mathrm{S}}[\mathrm{M} \Omega]$ & see figure 17 & $\begin{array}{c}2.91 \\
\text { (only for the series) }\end{array}$ & - & - & $\begin{array}{c}2.87 \text { (series) } \\
2.87(\mathrm{SP}) \\
-(\text { parallel })\end{array}$ \\
\hline$R_{1}[\mathrm{k} \Omega]$ & 4.64 & $\begin{array}{c}4.64 \text { (parallel) } \\
\text { changes (series) }\end{array}$ & 4.64 & 4.64 & $\begin{array}{c}4.64 \text { (parallel) } \\
7.24 \text { (series) }\end{array}$ \\
\hline$R_{2}[\mathrm{k} \Omega]$ & 4.64 & $\begin{array}{l}\text { changes (parallel) } \\
4.64 \text { (series) }\end{array}$ & 4.51 & 4.64 & $\begin{array}{c}R_{1 \mathrm{~s}}=15.18, R_{1 \mathrm{p}}=4.64(\mathrm{SP}) \\
4.64 \text { (parallel) } \\
4.64 \text { (series) }\end{array}$ \\
\hline$\hat{C}[\mathrm{nF}]$ & 44.35 & $\begin{array}{c}20.30 \text { (parallel) } \\
63.65 \text { (series) }\end{array}$ & 20.40 & 49.10 & $\begin{array}{c}R_{2 \mathrm{~s}}=4.64, R_{2 \mathrm{p}}=4.64(\mathrm{SP}) \\
19.82(\text { parallel }) \\
58.05(\text { series }) \\
\hat{C}_{\mathrm{s}}=56.90, \hat{C}_{\mathrm{p}}=19.82(\mathrm{SP})\end{array}$ \\
\hline$\hat{R}[\mathrm{M} \Omega]$ & 0.92 & $\begin{array}{l}\text { changes (for parallel RC) } \\
\text { changes (series) }\end{array}$ & - & 2.60 & $\begin{array}{c}-(\text { parallel }) \\
1.79(\text { series }) \\
0.86(\mathrm{SP})\end{array}$ \\
\hline
\end{tabular}

equation (59)) in order to verify if an increased value of $\left|R_{\mathrm{eq}}\right|$ was actually able to bring the behaviour of $Z_{\mathrm{rs}}$ closer and closer to that of $Z_{\text {is. }}$. Figure 17 shows the relation between $\tau_{e}$ (see equation (25)) and $H_{\mathrm{sc}} / H_{1}^{\max }$ (it takes the value of $A_{\mathrm{dB}}$ if $\tau_{e}=\tau_{e}^{\mathrm{opt}}$, see equations (42) and (45)) for the IC (i.e. $\left.R_{\text {eq }}=-\infty\right)$ and with some other values of $R_{\text {eq }}$ for the first mode of table 7 . The markers are related to the experimental results. The figure shows that a proper choice of $R_{\mathrm{S}}$ is actually able to make $Z_{\mathrm{rs}}$ similar to $Z_{\mathrm{is}}$ and that RCs tend to behave like ICs when $\left|R_{\text {eq }}\right|$ is increased.

Figure 17 also shows that all the curves of the plot are close to the blue solid curve, which represents the behaviour of the IC, when $\tau_{e}$ is high (over about $3.5 \cdot 10^{-3} \mathrm{~s}$ ). Therefore, this means that RCs and ICs provide similar attenuation values when $R_{\mathrm{sh}}$ and $\tau_{e}$ are high, as already mentioned in section 5.3. Hence, RCs in this area can be well described by the approach presented for ICs, even if $R_{\mathrm{S}}$ is not used. Conversely, the addition of $R_{\mathrm{S}}$ is useful when $R_{\mathrm{sh}}$ is low. Indeed, when $R_{\mathrm{sh}}$ is low, the distance between the curve related to the IC (solid blue curve) and the curves related to the RC (e.g. red dashed curve) increases with decreasing the value of $\left|R_{\text {eq }}\right|$, thus providing evidence of the different behaviour between RCs and IC (see the left-hand part of figure 17).

Some further facts are worth mentioning. There are two ways of letting the RC behave as an IC: add a compensation resistance $R_{\mathrm{S}}$ to bring the equivalent resistance $R_{\text {eq }}$ to $-\infty$ (see equation (59) and figure 12(c)), or use high values of $\hat{R}$ (see equation (55)). The latter solution proves to be unfeasible. Indeed, $\hat{R}$ is needed to let the bias currents in the OP-AMP flow to the ground and its value cannot be increased too much. Values of $\hat{R}$ higher than $3 \mathrm{M} \Omega$ generated in the tests bias currents leading the OP-AMP output to saturation. Therefore, the addition of the compensation resistance $R_{\mathrm{s}}$ was necessary. Nevertheless, the addition of $R_{\mathrm{s}}$ can cause a problem as well: high values of the equivalent resistance $\left|R_{\text {eq }}\right|$ 


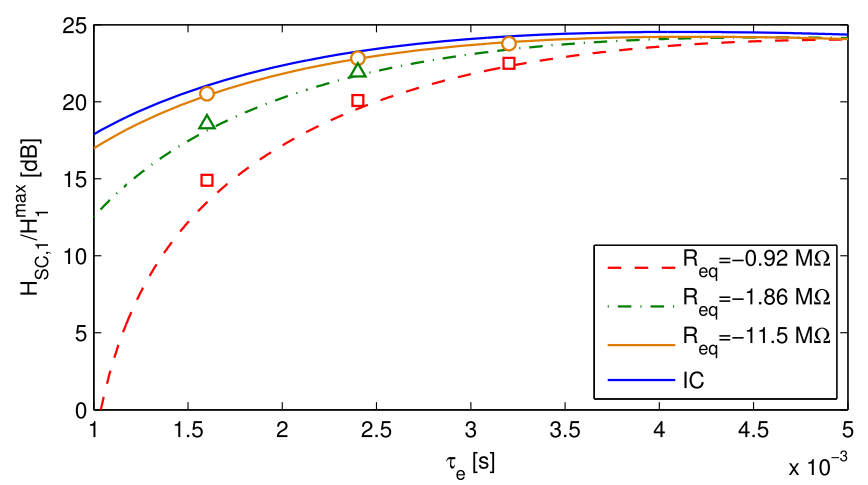

Figure 17. The trend of $H_{\mathrm{sc}} / H_{1}^{\max }$ as a function of $\tau_{e}$ for the first mode of the experimental set-up for different values of $R_{\text {eq }}$. The lines are obtained by means of the analytical model, the circles are the experimental points relating to the case of $R_{\mathrm{eq}}=-11.5 \mathrm{M} \Omega$

$\left(R_{\mathrm{S}}=1.00 \mathrm{M} \Omega\right)$, the triangles to the case of $R_{\mathrm{eq}}=-1.86 \mathrm{M} \Omega$

$\left(R_{\mathrm{s}}=1.82 \mathrm{M} \Omega\right)$ and the squares to the case of $R_{\mathrm{eq}}=-0.92 \mathrm{M} \Omega$

(which is the case without using $R_{\mathrm{s}}$ ). Refer to table 7 (test 1 column) for the value of $\tilde{R}$.

(achieved using $R_{\mathrm{S}}$ ) can lead to a significant DC voltage offset in the OP-AMP output. This phenomenon reduces the maximum possible dynamic output of the OP-AMP before saturation. Nonetheless, in the experiments carried out, if a value of $\left|R_{\text {eq }}\right|$ lower than about $100 \mathrm{M} \Omega$ was used, this offset often resulted equal to one or few volts.

Furthermore, the use of the compensation resistance $R_{\mathrm{s}}$ is needed when $R_{\text {sh }}$ is low (see previously in this section) and the attenuation provided by the shunt circuit is focused at high frequency. In such cases, if $R_{\mathrm{S}}$ is not used, the lowest eigenfrequencies tend to be amplified because of the presence of $\hat{R}$. This makes the output of the OP-AMP oscillate at low frequency with large amplitudes, thus reducing the maximum possible dynamic output of the OP-AMP usable for vibration attenuation before saturation. Hence, both the situations (i.e. the use or the avoidance of $R_{\mathrm{S}}$ ) lead to a decrease in the maximum possible dynamic output of the OP-AMP usable for vibration attenuation. Often, the DC offset due to $R_{\mathrm{s}}$ is lower than the amplitude of the low-frequency oscillations and thus the introduction of the compensation resistance $R_{\mathrm{S}}$ is advantageous.

Another reason for adding the compensation resistance $R_{\mathrm{s}}$, despite the DC offset issue, is that the stability limits are improved with respect to the case of the RCs because they approach those of the ICs as $\left|R_{\text {eq }}\right|$ tends to $\infty$. Indeed, the stability limits for RCs are more restrictive than for ICs (see appendix A).

Figure 17 also shows that the analytical model presented in $\mathrm{A}$, developed for $\mathrm{RCs}$, is reliable and accurate. Indeed, the experimental points are very close to the numerical model (i.e. curves with finite values of the equivalent resistance $R_{\mathrm{eq}}$ ). The accuracy of this model will be further remarked upon in section 6.3 .

\subsection{Validation of strategies for vibration reduction}

This subsection aims at validating the single-mode and multimode optimisation strategies (section 4) and the stability limits (section 2.6) for the parallel and series configurations.
The parallel configuration was built using both an IC and an RC for the negative capacitance. Both the layouts proved to be reliable. Indeed, if an IC is used, $R_{\mathrm{sh}}$-together with the piezoelectric patch capacitance-acts as a high-pass filter allowing the bias currents to flow to the ground. Hence, only IC will be discussed here. Furthermore, both type A and type B layouts were tested (see figure 11). Layout B proved to be more reliable so that all the tests shown further on in the section refer to such a configuration.

As for the series configuration, the IC (both type A and B) configurations led to the saturation of the OP-AMP and thus to instability because of the DC output component and bias currents. Therefore, the circuits tested in these experiments were built with an RC both with and without the addition of the compensation resistance $R_{\mathrm{S}}$ (see section 6.2). Both type AR and type BR layouts were tested. No significant differences were noticed in the behaviour of the two circuits. Therefore, the tests shown further on in this section refer only to type AR. Note that no further analyses were carried out for the differences between types $\mathrm{A}$ and $\mathrm{B}$ and between types $\mathrm{AR}$ and $\mathrm{BR}$, because this was out of the scope of the paper and is related to a deeper analysis of electronic issues.

The single-mode optimisation presented in section 4 was validated for all of the first four bending modes, whose parameters are shown in table 7 (test 2 column). Different cases were considered by changing the $\mathrm{NC}$ value. We took into account the values of the NC both close to and far from the stability limits (i.e. $C_{0}=33.26 \mathrm{nF}$ for the series and $C_{\infty}=25.9 \mathrm{nF}$ for the parallel, see section 6.1). The optimal value of $R_{\mathrm{sh}}$ for a given mode was calculated for each configuration by using equations (25) and (40) and the FRF was measured. Then, $A_{\mathrm{dB}}$ was calculated with the peak amplitude at the targeted resonance, with equation (42). Figure 18 shows the value of $A_{\mathrm{dB}}$ as a function of $C_{\mathrm{n}} / C_{\mathrm{p}}\left(C_{1} / C_{\mathrm{p}}\right.$ for the parallel configuration; $C_{2} / C_{\mathrm{p}}$ for the series configuration; $C_{\mathrm{p}}=30.5 \mathrm{nF}$ ), for the first four bending modes, and enables us to compare the experimental results with the theory predicted by equation (43) (solid lines in figure 18). There is a good agreement between the theory and experiments. The only case in which the accuracy decreases is related to the control of the second mode with the NC in series (i.e. discrepancies between the experiments and analytical expectations of $2 \mathrm{~dB}$ on average, see figure 18(b)); however, in this case the signal-to-noise ratio of the structural response signal was found to be poor.

As for the validation of the stability limits, it is worth highlighting some facts. The instability limits found in section 2.6 and appendix A are related to the dynamic instability of the EMS (i.e. the classical instability due to poles with positive real parts), which were derived considering the OP-AMP as ideal. In practice, another kind of instability can arise which is related to the non-linear behaviour of the OP-AMP. Indeed, when the voltage induced at the terminal of the NC is high, the output of the OP-AMP can saturate, leading to the instability of the EMS. The closer $C_{\mathrm{n}}$ is to the stability limit, the higher the voltage induced at the terminal of the $\mathrm{NC}$ is, and thus such a problem arises when the value of $C_{\mathrm{n}} / C_{\mathrm{p}}$ approaches 1 . Therefore, it is important to 

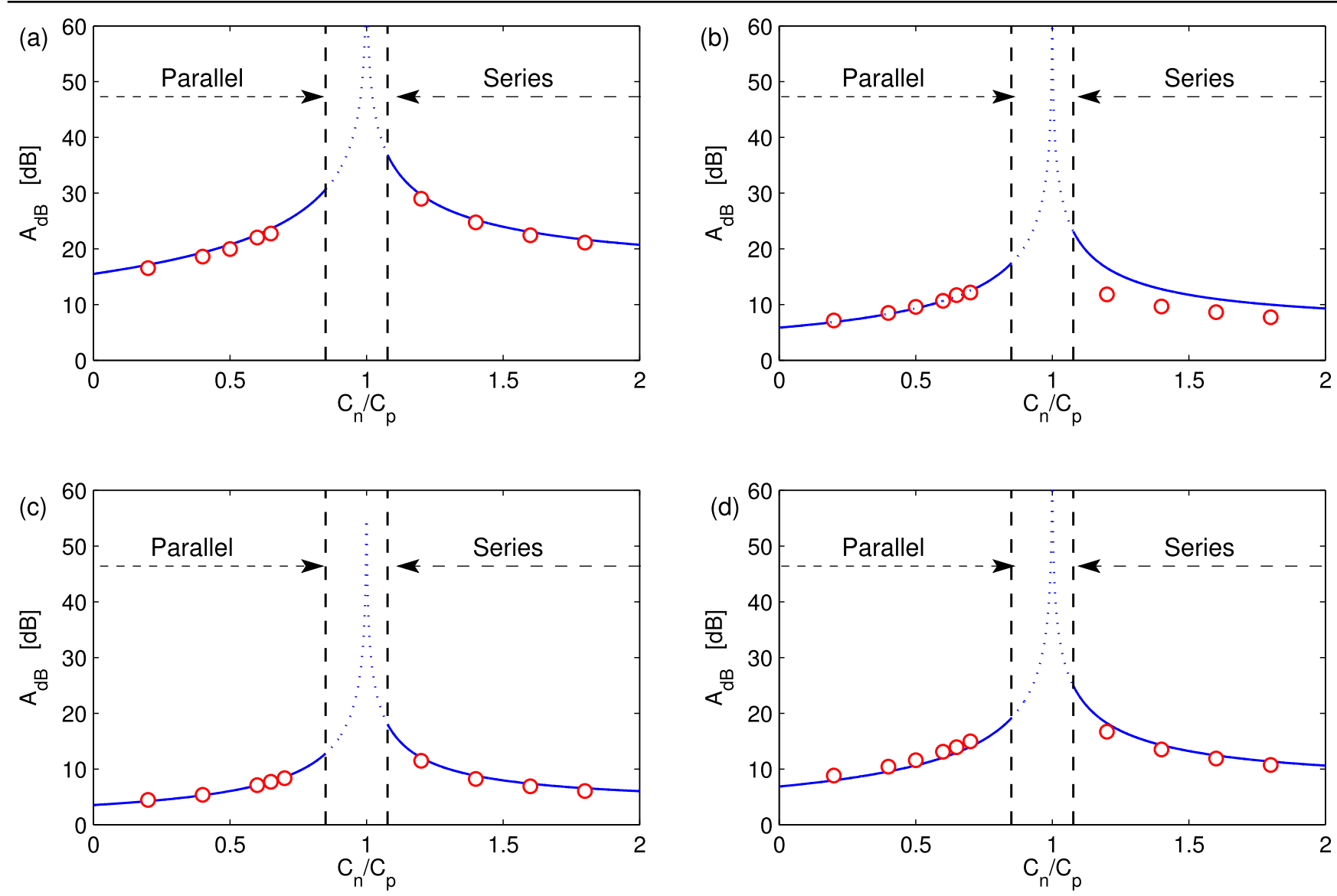

Figure 18. The trend of $A_{\mathrm{dB}}$ as a function of $C_{\mathrm{n}} / C_{\mathrm{p}}$ for the first mode (a), second mode (b), third mode (c) and fourth mode (d) of the piezoelectric beam. The lines are obtained by means of the analytical model, the circles are the experimental points. The vertical dashed lines correspond to the stability limits (i.e. $C_{\infty}$ for the line on the left and $C_{0}$ for the line on the right). The curves and circles on the left of $C_{\mathrm{n}} / C_{\mathrm{p}}=1$ are related to the parallel configuration and the curves and points on the right of $C_{\mathrm{n}} / C_{\mathrm{p}}=1$ are related to the series layout. For the experiments relating to test 2 of table 7, the series configuration was built using $R_{\mathrm{S}}$ (see section 6.2).

distinguish between these two cases (i.e. dynamic instability and OP-AMP saturation) to validate the stability limits of section 2.6.

As for the parallel configuration, the dynamic instability was found for a value of $C_{1}$ equal to $22.9 \mathrm{nF}$ (which corresponds to $\left.\beta_{1}=C_{1} / C_{\mathrm{p}}=0.75\right)$, which is lower than the theoretical limit (i.e. $25.9 \mathrm{nF}, \beta_{1}^{\text {inst }}=C_{\infty} / C_{\mathrm{p}}=0.85$ ). Nonetheless, it is hard to have a very accurate estimation of $C_{\infty}$ (see section 6.1 and [25]) and the value of the capacitance measured at $100 \mathrm{kHz}$ is just an estimation. Furthermore, the modes which become unstable with the parallel configuration are those at high frequency (section 2.6), where the model can start to become less accurate. For all these reasons, the experimental limit (i.e. $22.9 \mathrm{nF}$ ) can be considered as a satisfactory validation of the condition given in section 2.6. The experimental value of $C_{1} / C_{\mathrm{p}}$ closest to the stability limits shown in figure 18 is 0.65 , because instability phenomena due to saturation of the OP-AMP sometimes occurred for values of $C_{1} / C_{\mathrm{p}}$ between 0.65 and 0.75 .

As for the series configuration, dynamic instability did not occur until values of $\beta_{2}=C_{\mathrm{p}} / C_{2}$ lower than 0.88 . This result is in good agreement with the theoretical expectation for the stability limit: $\beta_{2}^{\text {inst }}=0.917$ (see equation (38)). The test with $\beta_{2}=0.88$ was not included in figure 18 because it was performed just for the first mode (for this test the experimental value of $A_{\mathrm{dB}}$ was found to be $1.5 \mathrm{~dB}$ lower than the theoretical expectation). We could not perform the same tests for modes 2 to 4 because the low value of $R_{\mathrm{sh}}$ required for optimal control, together with this $C_{2} / C_{\mathrm{p}}$ value, led to a significant non-linear behaviour of the OP-AMP and the piezoelectric patch.

Finally, figure 19 shows the experimental validation of the curves of $H_{i}^{\max }$ as a function of $\tau_{e}$ (i.e. equations (45) and (49a)) used for the graphical multi-modal optimisation strategy presented in section 4.4. The parallel NC for this test was built as mentioned at the beginning of this section (i.e. type B), while the series $\mathrm{NC}$ was built as in section 6.2 but without adding the compensation resistance $R_{\mathrm{s}}$. This choice allowed the authors to further validate the model for RCs presented in appendix A. The parameter values used in the tests are provided in table 7 (test 3 column for the parallel and test 4 column for the series). Again, the agreement between numerical expectations and experimental results is excellent.

The curves of figure 19 give us the occasion to show that the refined model for the piezoelectric patch capacitance, introduced in equations (8) and (9), allows us to better estimate the performance of the control action. Indeed, figure 20 shows the comparison between the curves describing 

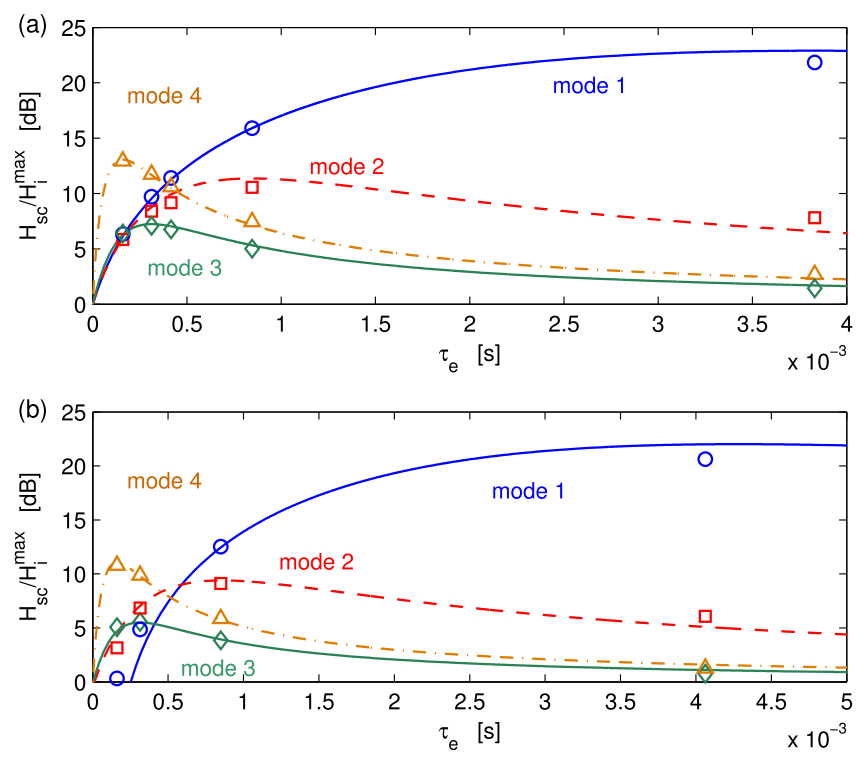

Figure 19. The trend of $H_{\mathrm{sc}} / H_{i}^{\max }$ as a function of $\tau_{e}$ for the parallel (a) and series (b) configurations, for the four modes of the experimental set-up. The lines are related to the analytical model (section 4.4) and the markers to experiments ('o': mode 1; ' $\square$ : mode 2 ; ' $\checkmark$ ': mode 3; ' $\triangle$ ': mode 4). See test 3 of table 7 for the parallel configuration and test 4 for the series configuration. The series configuration was built without using $R_{\mathrm{s}}$ (see appendix A).

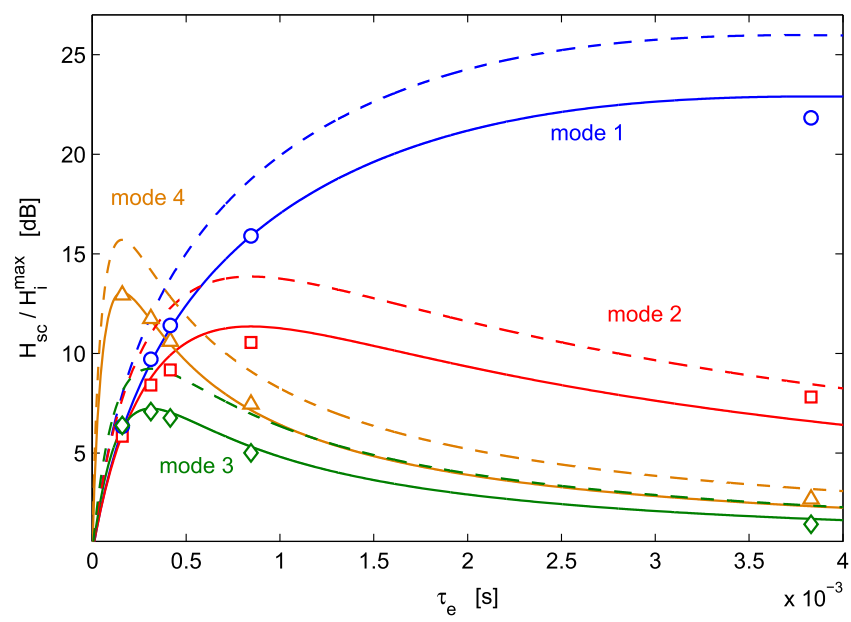

Figure 20. The trend of $H_{\mathrm{sc}} / H_{i}^{\max }$ as a function of $\tau_{e}$ for the parallel configuration, for the four modes of the experimental set-up. The solid lines are related to the analytical model using $C_{\mathrm{p} i}$ (equations (8) and (9)), the dashed lines to the analytical model using $C_{\infty}$ (equation (6)), and the markers to experiments ('o': mode 1; ' $\square$ ': mode 2 ; ' $\diamond$ ': mode 3 ; ' $\triangle$ ': mode 4 ).

$H_{\mathrm{sc}} / H_{i}^{\max }$ achieved using the refined model (solid lines in the figure) and the ones using $C_{\infty}$ (dashed lines) for the parallel case. The solid lines and the experimental points in figure 20 are the same lines and points of figure 19(a). The estimations provided by the refined model proved to be much more accurate.

\subsection{Tests with the SP configuration}

This subsection aims at testing the newly proposed SP configuration and comparing its attenuation performances with

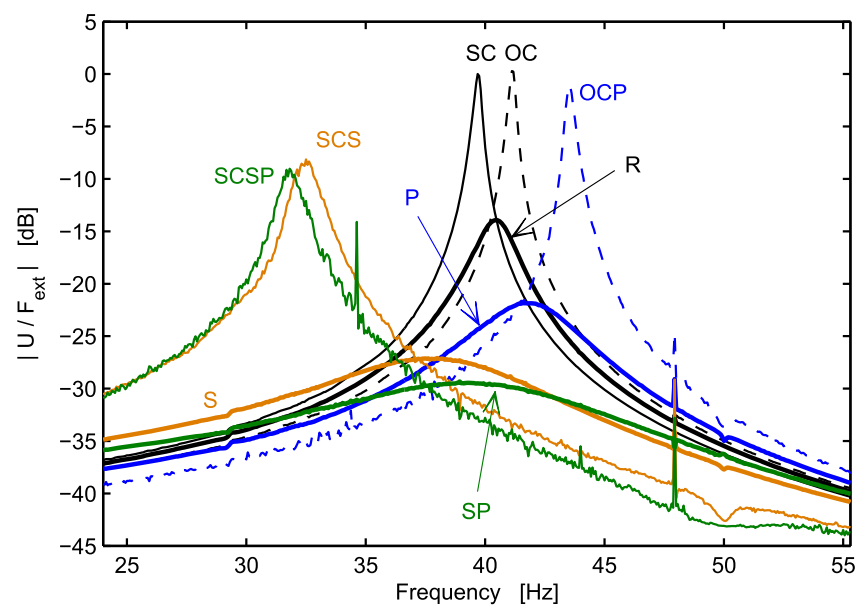

Figure 21. Experimental FRFs (displacement $U$ to force $F_{\text {ext }}$ ratio), around the first resonance of the EMS, for different configurations, analogous to figure 4 (except for the short circuit response of the SP configuration (SCSP) that is slightly distinct to SCS in experiments).

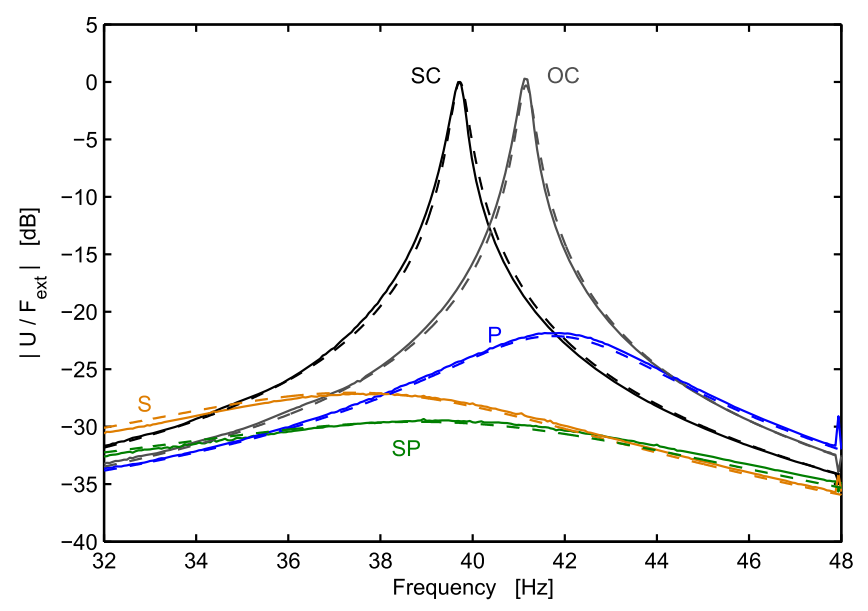

Figure 22. FRFs (displacement $U$ to force $F_{\text {ext }}$ ratio), around the first resonance of the EMS, for different configurations, analogous to figure 4. Comparison of experiments (solid lines) and theory (dashed lines).

those of traditional circuits. In particular, the SP layout was compared to a simple resistive shunt (i.e. no use of any $\mathrm{NC}$ ), the series configuration of the $\mathrm{NC}$ and the parallel layout of the NC. As for the SP circuit, the NC in parallel was built using an IC (layout B), while the NC in series was built with an RC (layout AR) with the addition of the compensation resistance $R_{\mathrm{s}}$ (see section 6.2). The circuit is displayed in figure 14 . The parameters used for these tests are provided in test 5 column of table 7 . Figure 21 shows the experimental FRFs of the EMS (zoomed on the first mode) in short-circuit, open-circuit and when an optimal resistive shunt is used for four different cases:

- with no $\mathrm{NC}$ in the shunt circuit;

- with an NC in parallel and $C_{1} / C_{\mathrm{p}}=\beta_{1}=0.65$

- with an $\mathrm{NC}$ in series and $C_{2} / C_{\mathrm{p}}=1.22$ (i.e. $\beta_{2}=0.82$ ) 


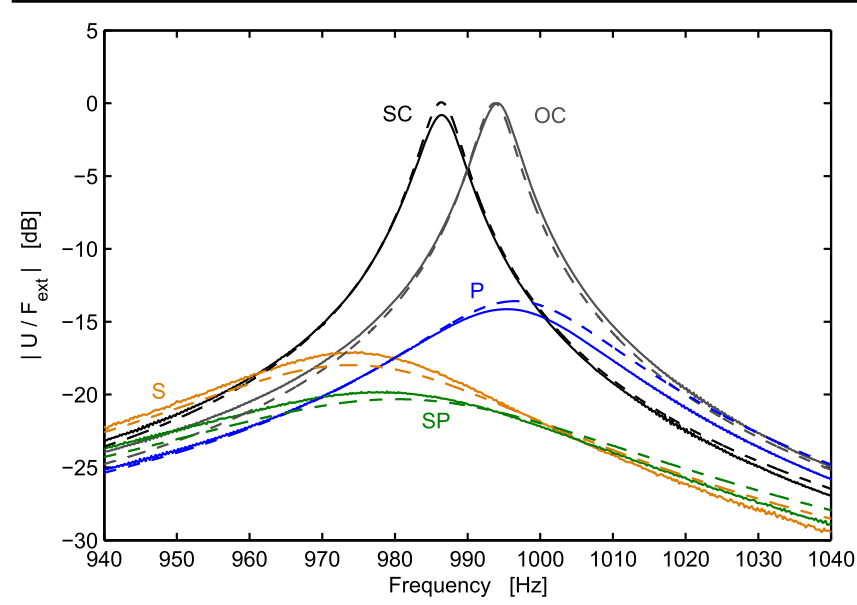

Figure 23. FRFs (displacement $U$ to force $F_{\text {ext }}$ ratio), around the fourth resonance of the EMS, for different configurations, analogous to figure 4. Comparison of experiments (solid lines) and theory (dashed lines).

- with two $\mathrm{NCs}$ in $\mathrm{SP}$ configuration with $\beta_{\mathrm{sp} 1}=\beta_{1}$ and $\beta_{\mathrm{sp} 2}=\beta_{2}$.

These values were chosen because they were those that provided at the same time the best attenuation and avoidance of both saturation and non-linearity for the parallel, series and SP configurations.

Figure 21 shows that the SP configuration is able to shift both the open- and short-circuit eigenfrequencies at the same time, thus allowing the attenuation performance to be improved. The maximum of the FRF of the SP with the optimal value of $R_{\text {sh }}$ (i.e. $R_{\text {opt }}$ ) is close to the intersection point of the corresponding open- and short-circuit FRFs, as expected, and the same occurs for all the configurations considered, in accordance with the analytical approach proposed (see section 4 and figure 4 ). It has been noticed that the SP tends to be unstable in short-circuit condition and becomes stable once a given value of $R_{\mathrm{sh}}$ is achieved. Such a value was $890.5 \Omega$ for the test case of figure 21 . This threshold value on $R_{\text {sh }}$ was found to get lower and lower once the values of $C_{1}$ and $C_{2}$ moved farther and farther from the stability limits.

Figure 22 shows the numerical FRFs corresponding to some of the experimental FRFs shown in figure 21, and the agreement is good, proving again the reliability of the analytical model presented herein. Moreover, the effectiveness of the SP is confirmed because it is able to improve the performance of the series configuration of about $2.4 \mathrm{~dB}$ and that of the parallel layout of about $7.8 \mathrm{~dB}$ on the first mode. Finally, figure 23 shows analogous results for the fourth resonance.

\section{Conclusion}

This paper has addressed several points regarding the theory and experiments on resistive shunt damping enhanced by the use of negative capacitances (NCs). The most original contributions to the topic are: the use of a novel layout based on the use of two NCs at the same time, able to improve the performance of the traditional NC layouts; the development of a refined multi-mode model for the electro-mechanical system (EMS); the single and multi-mode optimisation strategies as well as analytical formulas to foresee the vibration attenuation level; finally, the analytical treatment of the real circuits used to build the NC. In particular, a multi-mode theoretical model of the EMS has been proposed and reduced to a single degree of freedom in a refined manner, by carefully analysing the piezoelectric patch capacitance dependence on the frequency and using static corrections. This refined description of the patch capacitance is found to be mandatory when dealing with negative capacitance circuits, since it is directly linked to the dynamical stability of the system.

This model enables an analytical formalism to be developed that is common to all the treated resistive shunt layouts: the traditional one (without the NC), an NC in parallel to the piezoelectric patch, an NC in series, and a new configuration using two NCs in series and in parallel. It is first demonstrated that the effect of the NCs is to artificially increase the modal electro-mechanical coupling factors (MEMCF) of the structure by changing the piezoelectric patch capacitance viewed by the shunt; this makes it possible to define an enhanced MEMCF. Then, it is shown that the tuning and the single-mode control performances of the shunt rely only on the enhanced MEMCF and on the intrinsic damping factor of the EMS, in the same way as for traditional R- and RL-shunts. Since the R-shunt performances are increased by the NC, it is also shown that this kind of control leads to a significant broadband attenuation, whereas the usual modest performances of $\mathrm{R}$-shunts are reduced to a single resonance. The proposed new negative capacitance layout is shown to further increase the shunt performance. All these theoretical results are validated by experiments.

Furthermore, the paper explains how to optimise the shunt impedance for the purpose of multi-mode control, explains how to make RCs (i.e. the circuits usually used in practice to build NCs) behave like ICs (i.e. pure NCs) and even develops the analytical treatment of RCs.

\section{Acknowledgments}

The Structural Mechanics and Coupled Systems Laboratory (LMSSC-Cnam, Paris) and its head Jean-François Deü are gratefully thanked for the loan of the piezoelectric beam used in the experiments.

\section{Appendix A. Analytical treatment for real circuits in series}

Here, the equations governing the behaviour of the EMS with an $\mathrm{RC}$ in series are described. In this case, the circuit taken into consideration is that of figure 12(b). The impedance $Z_{\mathrm{rs}}$ of the shunt circuit is composed by $-C_{2},-\tilde{R}$ and $R_{\mathrm{sh}}$. Therefore, $Z_{\mathrm{rs}}$ can be expressed as in equation (57). Relying on equations $(22 a)-(c)$, taking into consideration that 
$V=-Z_{\mathrm{rs}} \dot{Q}$ and truncating the model to a single-degree-offreedom system (see equations (2a) and (9)), the following FRF is yielded:

$\frac{q_{i}}{F_{i}}=\frac{-\Omega^{2} C_{\mathrm{p} i} C_{2} \tilde{R} R_{\mathrm{sh}}+\mathrm{j} \Omega\left[C_{2} \tilde{R}+C_{\mathrm{p} i}\left(-\tilde{R}+R_{\mathrm{sh}}\right)\right]+1}{\Omega^{4} C_{\mathrm{p} i} C_{2} \tilde{R} R_{\mathrm{sh}}-\mathrm{j} \Omega^{3} A_{3}-\Omega^{2} A_{2}+\mathrm{j} \Omega A_{1}+\omega_{i}^{2}}$

where:

$$
\begin{gathered}
A_{1}=2 \xi_{i} \omega_{i}+\omega_{i}^{2} C_{2} \tilde{R}+\left(\omega_{i}^{2} C_{\mathrm{p} i}+\chi_{i}^{2}\right)\left(-\tilde{R}+R_{\mathrm{sh}}\right) \\
A_{2}=2 \xi_{i} \omega_{i} C_{2} \tilde{R}+2 \xi_{i} \omega_{i} C_{\mathrm{p} i}\left(-\tilde{R}+R_{\mathrm{sh}}\right) \\
+\omega_{i}^{2} C_{\mathrm{p} i} C_{2} \tilde{R} R_{\mathrm{sh}}+\chi_{i}^{2} C_{2} \tilde{R} R_{\mathrm{sh}}+1 \\
A_{3}=C_{2} \tilde{R}+C_{\mathrm{p} i}\left(-\tilde{R}+R_{\mathrm{sh}}\right)+2 \xi_{i} \omega_{i} C_{\mathrm{p} i} C_{2} \tilde{R} R_{\mathrm{sh}}
\end{gathered}
$$

If we apply the Routh-Hurwitz criterion [16] to equation (A.1), the stability of the EMS with $R_{\mathrm{sh}}=0$ (i.e. new short-circuit condition) is assured if the following equations are satisfied:

$$
\begin{aligned}
C_{2} & >C_{\mathrm{p} i}+\frac{\chi_{i}^{2}}{2 \omega_{i}^{2}}-\frac{\xi_{i}}{\omega_{i} \tilde{R}} \\
& +\frac{\sqrt{\left(2 \xi_{i} \omega_{i}-\chi_{i}^{2} \tilde{R}\right)\left[2 \omega_{i}\left(\xi_{i}^{2}-1\right)-\chi_{i}^{2} \xi_{i} \tilde{R}\right]}}{2 \omega_{i}^{2} \tilde{R} \sqrt{\xi_{i}}}, \\
& \text { if } \tilde{R}>\frac{2 \xi_{i} \omega_{i}}{\chi_{i}^{2}}
\end{aligned}
$$

or

$$
C_{2}>C_{\mathrm{p} i}, \quad \text { if } \tilde{R} \leqslant \frac{2 \xi_{i} \omega_{i}}{\chi_{i}^{2}}
$$

If all the modes of the structure are taken into consideration, the stability conditions of equations (A.5) and (A.6) must be satisfied for all the modes of the EMS. This is a sufficient condition for stability. The use of $R_{\text {sh }} \neq 0$ makes the stability condition difficult to be calculated analytically. There are two ways to calculate the stability conditions:

- numerical simulations, which show that the stability conditions with $R_{\mathrm{sh}} \neq 0$ tend to be less restrictive if compared to the conditions of equations (A.5) and (A.6). Therefore, the condition of equations (A.5) and (A.6) can yet be used to guarantee the stability of the EMS (actually the effect of $R_{\text {sh }}$ is that of an added damper);

- if an accurate value of the stability limit is needed, the following system of equations must be solved for each mode to find the stability conditions thanks to the Routh-
Hurwitz criterion:

$$
\left\{\begin{array}{l}
A_{3}>0 \\
A_{2}>0 \\
A_{1}>0 \\
\frac{A_{1}}{C_{\mathrm{p} i} C_{2} \tilde{R} R_{\mathrm{sh}}}<\frac{A_{3} A_{2}}{\left(C_{\mathrm{p} i} C_{2} \tilde{R} R_{\mathrm{sh}}\right)^{2}} \\
\frac{\omega_{i}^{2}}{C_{\mathrm{p} i} C_{2} \tilde{R} R_{\mathrm{sh}}}<\frac{\left[A_{1} A_{2} A_{3} /\left(C_{\mathrm{p} i} C_{2} \tilde{R} R_{\mathrm{sh}}\right)^{3}\right]-\left[A_{1}^{2} /\left(C_{\mathrm{p} i} C_{2} \tilde{R} R_{\mathrm{sh}}\right)^{2}\right]}{\left(A_{3} /\left(C_{\mathrm{p} i} C_{2} \tilde{R} R_{\mathrm{sh}}\right)\right)^{2}}
\end{array}\right.
$$

Once the stability conditions are known, the optimal value of $R_{\mathrm{sh}}$ can be derived analytically. Relying on the FRF of equation (A.1) and following the same procedure discussed in section 4.4, the relation between a fixed value of $R_{\mathrm{sh}}$ and $\omega_{i}^{\mathrm{r}}$ can be found. This relation is expressed by a ninth order equation and a closed formulation for its solution cannot be found. The expression of the equation is quite long and thus we have not added it to the paper. Nevertheless, the reader can find it easily by using the same approach presented in section 4.4 (see equations (44) and (45)). Such an equation provides nine solutions (one of them is the trivial solution equal to zero). Then, the physical solution must be looked for so that this procedure can be used to draw the curve linking $R_{\text {sh }}$ and $H_{i}^{\max }$ and thus mono- and multi-modal optimisations can still be carried out.

\section{References}

[1] Hagood N and von Flotow A 1991 Damping of structural vibrations with piezoelectric materials and passive electrical networks J. Sound Vib. 146 243-68

[2] Thomas O, Ducarne J and Deü J 2012 Performance of piezoelectric shunts for vibration reduction Smart Materials and Structures 21015008

[3] de Marneffe B and Preumont A 2008 Vibration damping with negative capacitance shunts: theory and experiment Smart Materials And Structures 17035015

[4] Behrens S, Fleming A J and Moheimani S O R 2003 A broadband controller for shunt piezoelectric damping of structural vibration Smart Mater. Struct. 12 18-28

[5] Date M, Kutani M and Sakai S 2000 Electrically controlled elasticity utilizing piezoelectric coupling J. Appl. Phys. 87 863-8

[6] Park C H and Baz A 2005 Vibration control of beams with negative capacitive shunting of interdigital electrode piezoceramics J. Vib. Control 11 331-46

[7] Manzoni S, Moschini S, Redaelli M and Vanali M 2012 Vibration attenuation by means of piezoelectric transducer shunted to synthetic negative capacitance J. Sound Vib. 331 4644-57

[8] Kodejška M, Mokrý P, Linhart V, Václavik J and Sluka T 2012 Adaptive vibration suppression system: An iterative control law for a piezoelectric actuator shunted by a negative capacitor IEEE Transactions on Ultrasonics, Ferroelectrics, and Frequency Control 59 2785-96

[9] Beck B S, Cunefare K A and Collet M 2013 The power output and efficiency of a negative capacitance shunt for vibration control of a flexural system Smart Materials and Structures 22065009

[10] Beck B S, Cunefare K A and Collet M 2014 Response-based tuning of a negative capacitance shunt for vibration control J. Intell. Mater. Syst. Struct. 25 1585-95 
[11] Berardengo M, Cigada A, Manzoni S and Vanali M 2015 Vibration control by means of piezoelectric actuators shunted with LR impedances: performance and robustness analysis Shock and Vibration 2015704265

[12] Ducarne J, Thomas O and Deü J 2012 Placement and dimension optimization of shunted piezoelectric patches for vibration reduction $J$. Sound Vib. 331 3286-303

[13] Thomas O, Deü J-F and Ducarne J 2009 Vibration of an elastic structure with shunted piezoelectric patches: efficient finite-element formulation and electromechanical coupling coefficients Int. J. Numerical Methods in Engineering 80 235-68

[14] Preumont A 2011 Vibration Control of Active Structures 3rd ed (Berlin: Springer)

[15] Horowitz P and Hill W 1989 The art of electronics 2nd (Cambridge: Cambridge University Press)

[16] Gopal M 2002 Control Systems: Principles and Design 2nd edn (New Delhi: Tata McGraw-Hill)

[17] Burns R 2001 Advanced Control Engineering (Oxford: Butterworth-Heinemann)
[18] den Hartog J 1956 Mechanical Vibrations (New York: McGraw Hill)

[19] Snowdon J 1968 Vibration and Shock in Damped Mechanical Systems (New York: Wiley)

[20] Ducarne J, Thomas O and Deü J-F 2010 Structural vibration reduction by switch shunting of piezoelectric elements: modelling and optimization Journal of Intelligent Materials Systems and Structures 21 797-816

[21] Cardano G 1993 The Rules of Algebra (Ars Magna) (New York: Dover) 1545 published

[22] Moheimani S and Fleming A 2006 Piezoelectric Transducers for Vibration Control and Damping (London: Springer)

[23] Thomas O, Touzé C and Chaigne A 2003 Asymmetric nonlinear forced vibrations of free-edge circular plates. Part II: experiments J. Sound Vib. 265 1075-101

[24] Texas Instruments, 2010 OPA445, High Voltage FET-Input Operational Amplifier (http://ti.com/product/opa445)

[25] IEEE, 1988 An American National Standard-IEEE Standard on Piezoelectricity The Institute of Electrical and Electronics Engineers Inc 